\title{
Assessment of deep demersal fish fauna diversity of the Colombian Caribbean Sea
}

\author{
CAmilo B. García* and Jorge M. GamboA \\ Departamento de Biología, Universidad Nacional de Colombia, Carrera 45 \# 26-85, Bogotá, Colombia
}

Marine and

Fishery Sciences

MAFIS

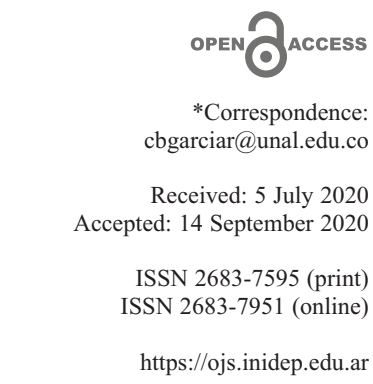

Journal of the Instituto Nacional de Investigación y Desarrollo Pesquero (INIDEP)

This work is licensed under a Creative Commons AttributionNonCommercial-ShareAlike 4.0 International License

\begin{abstract}
We compiled georeferenced records of deep demersal fishes from the Colombian Caribbean Sea in order to assess the level of survey coverage and geographic completeness of species richness inventories at a scale of $15 \mathrm{~min}$ by $15 \mathrm{~min}$ cells, in view of threats from fishing and oil and natural gas exploration. We identified a rich fauna with a minimum of 362 species registered. Areas with high observed and predicted species richness were identified. Survey coverage and geographic richness completeness resulted in being deficient with no cell reaching the status of wellsampled spatial unit, being $83 \%$ of the Colombian Caribbean Exclusive Economic Zone bottoms unexplored, particularly depths beyond $1,000 \mathrm{~m}$. A plea is made for renewed survey efforts with a focus on the protection of the Colombian Caribbean deep-sea biota.
\end{abstract}

Key words: Colombian Caribbean, deep fishes, records, soft-bottoms, species richness.

Evaluación de la diversidad de la fauna de peces demersales profundos del Mar Caribe colombiano

RESUMEN. Se recopilaron registros georreferenciados de peces demersales profundos del Mar Caribe colombiano con el fin de evaluar el nivel de cobertura de la prospección y la integridad geográfica de los inventarios de riqueza específica a una escala de celdas de 15 min por 15 min, en vista de las amenazas de la pesca y la explotación de petróleo y gas. Identificamos una rica fauna con un mínimo de 362 especies registradas. Se identificaron áreas con alta riqueza específica observada y predicha. La cobertura de los registros y la completitud geográfica de la riqueza resultaron ser deficientes sin que ninguna celda alcanzara el estado de unidad espacial bien muestreada, estando el 83\% de los fondos de la Zona Económica Exclusiva del Caribe colombiano sin explorar, en particular aquellas profundidades superiores a los $1.000 \mathrm{~m}$. Se hace un llamamiento para renovar los esfuerzos de prospección con un enfoque en la protección de la biota de aguas profundas del Caribe colombiano.

Palabras clave: Caribe colombiano, peces de fondo, registros, fondos blandos, riqueza específica.

\section{INTRODUCTION}

With the migration of the fishing frontier into deeper waters and the offshore search for oil and natural gas it is now more important than ever to improve the knowledge on the deep sea biota, particularly with regard to 
fishes, since they are candidates for exploitation (e.g., Páramo et al. 2017; Grijalba-Bendeck et al. 2019) or might be affected as bycatch (e.g., Páramo et al. 2012). In Colombian Caribbean waters deep sea research has been sporadic, but three main periods can be distinguished. In the late sixties and early seventies during the past century, research vessels like the Oregon, Oregon II (U.S. Fish and Wild Life Service) and Pillsbury (University of Miami, U.S.A.), as well as several others, conducted trawling both on the continental shelf and notably on the continental slope, plus some trawls occurred at depths beyond $1,000 \mathrm{~m}$. Later on, from the early part of the 20th century, interest in continental slope fauna gained momentum with the Macrofauna I and II campaigns that trawled down to a depth of $500 \mathrm{~m}$ (see for instance Saavedra-Díaz et al. 2000; Roa-Varón et al. 2003; Saavedra-Díaz et al. 2004; Roa-Varón et al. 2007). A decade later in the context of exploration for oil and natural gas, demersal fish samples were taken in deeper waters around $800 \mathrm{~m}$ (ANH I and II campaigns, Polanco et al. 2010). In searching for new fishery resources, Páramo et al. (2011) conducted trawling at depths between 200 and $550 \mathrm{~m}$ during years 2009 and 2010. Since then, to our knowledge, no mayor deep sea trawling surveys have been undertaken in Colombian Caribbean waters.

Paramount in diversity studies (and with a focus on conservation) is the characterization of the geographic distribution of diversity, which goes hand in hand with the assessment of the completeness of sampling of surveys, i.e., whether an asymptote in the species accumulation curve has been reached (Soberón et al. 2007; Hortal et al. 2015). The composition of species lists is necessary but not sufficient for effective diversity management and conservation. In this study we assess the level of survey coverage and geographical completeness of species richness inventories by constructing a spatialized estimate of deep soft-bottom demersal fish fauna diversity in Colombian Caribbean waters, including the San
Andrés and Providencia archipelago and in the process we highlight areas that have been welland poorly surveyed and those that have never been visited.

\section{MATERIALS AND METHODS}

Georeferenced records of demersal deep fishes were obtained from Polanco (2015), García and Armenteras (2015, see sources cited), García (2017a), Acero et al. (2018), Polanco et al. (2019) and the Global Biodiversity Information Facility (GBIF 2020). Only records with associated depth registers deeper than $200 \mathrm{~m}$ were included. Validity of taxonomic names was checked against the Catalog of Fishes (Fricke et al. 2019). A database was constructed with the software ModestR (García-Roselló et al. 2013) which allowed further filtering in order to lessen redundancy in the geographic records. Because our interests focused on Colombian Caribbean waters, records in our sources outside the current Colombian Exclusive Economic Zone (EEZ, obtained from Flanders Marine Institute 2019) were excluded.

ModestR produces files directly usable for the module KnowBR (Lobo et al. 2018; Guisande and Lobo 2019) of the application RWizard (Guisande et al. 2014) designed to conduct a search of both well and poorly surveyed spatial units. The study area, i.e. the Colombian Caribbean EEZ, was divided into spatial cells of size $15 \mathrm{~min}$ (circa $28 \mathrm{~km}$ ) by $15 \mathrm{~min}$. We chose this size as being consistent with previous similar analyses (García 2017b, 2018). Further settings were as follow: curve = 'Rational' (Ratkowski 1990) one of the options for adjusting a function to the accumulation of species with records that function as surrogates for effort (Lobo 2008; Lobo et al. 2018); estimator $=1$, meaning that we constructed the species accumulation curve using the formula from Ugland et al. (2003); cutoff $=1$, meaning that if the quotient 
between number of records and number of species is 1 in a given spatial unit it is considered non-informative and completeness is not calculated; cutoffCompleteness $=0$, meaning that if the value of completeness is below this value of completeness is not calculated and cutoffSlope $=$ 1 , meaning that if the final slope of the accumulation curve is higher than this value, completeness is not calculated.

We used the function SurveyQ (survey quality) of KnowBR to depict well- and poorly-surveyed equal area cells geographically. Default values were used. For well sampled spatial units a slope $<0.02$, completeness $>90 \%$ and $\mathrm{R} / \mathrm{S}$ (records over species) $>15$. For poorly sampled cells a slope $>0.3$, completeness $<50 \%$ and $\mathrm{R} / \mathrm{S}<3$.

\section{RESULTS}

After filtering, we included a total of 362 species and 6,211 records in the database, these being the basis of the analysis (Appendix 1). A map of the study area, records and the shape of the Parque Nacional Natural Corales de Profundidad (http://www.parquesnacionales.gov.co) is shown in Figure 1. The bulk of the records were located between $200 \mathrm{~m}$ and $1,000 \mathrm{~m}$ representing $98 \%$ of all records. In turn, records between $200 \mathrm{~m}$ and $300 \mathrm{~m}$ represented $52 \%$ of all records. Apart from a couple of records in the San Andrés and Providencia archipelago, sampling was concentrated on

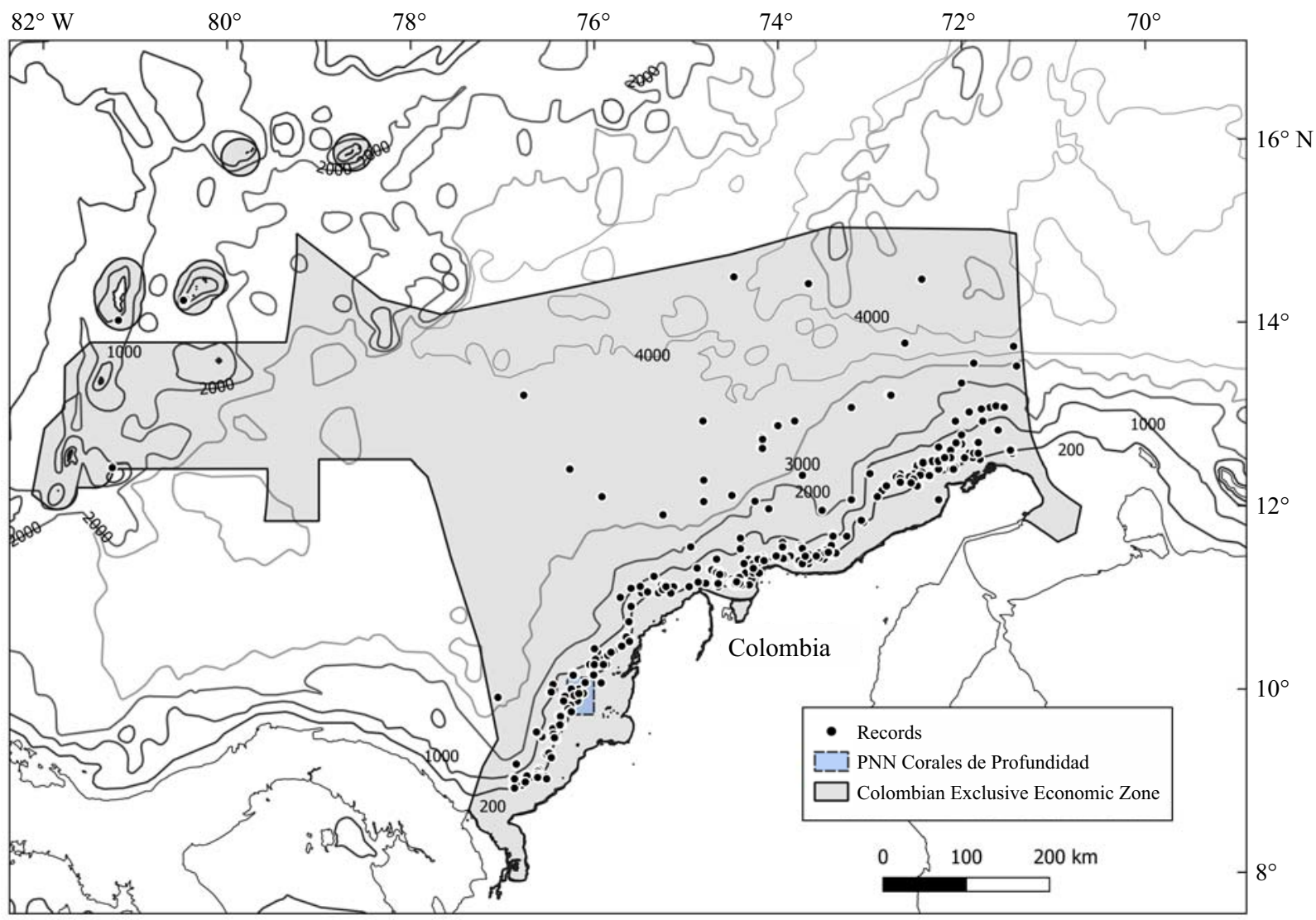

Figure 1. Deep demersal fishes record locations inside the Colombian Caribbean Exclusive Economic Zone and Parque Nacional Natural Corales de Profundidad. 
the continental slope with a few records from the abyssal plains (Figure 1). Table 1 shows those species with more than 100 records in the database.

The division of the study area in equal area cells (15 $\mathrm{min}$ by $15 \mathrm{~min}$ ) resulted in 85 cells. Records from each cell revealed some areas where sampling was concentrated. The Gulf of Salamanca, Guajira, Palomino and mixed coralline bottoms showed cells with an elevated number of records while most of the cells received fewer records (Figure 2). Taking into account that cells depicted were the ones with at least one record, it was clear that most of the study area had never been visited (Figure 2). The Colombian Caribbean EEZ below $200 \mathrm{~m}$ amounts to circa $385,000 \mathrm{~km}^{2}$ while the added cell area is $65,072 \mathrm{~km}^{2}$, so around $83 \%$ of the deep sea bottom has never been visited. The scarcity of records resulted in 31 out of the 85 cells for which completeness could not be calculated as the R/S quotient was one. Not surprisingly, the observed richness (Figure 3 ) closely followed the distribution pattern of records in cells with a correlation of 0.9 (Pearson index, $\mathrm{p}<0.001$ ). The Darien area is added as harboring a high number of demersal fish species (Figure 3).
Completeness ranged from $6.9 \%$ to $76.9 \%$ in cells with $75.9 \%$ of them (41 out of 54 informative cells) showing completeness above $50 \%$; but notice that no cell reached $100 \%$ completeness (Figure 4). The ten cells showing completeness above $70 \%$ were well- distributed along the coast line (Figure 4), suggesting that the survey effort was not spatially biased in terms of completeness.

No cell attains the status of 'high quality survey' (Figure 5); but most cells were labeled as 'fair quality survey' cells with a number of interdispersed 'poor quality survey' cells that is in line with the image in Figure 4.

\section{DISCUSSION}

Our focus on the current Colombian EEZ caused 32 species and associated records to be dropped from the database (Appendix 2). These records are located to the west of the San Andrés and Providencia archipelago and offshore Panama outside of Colombian EEZ. Thus, it is doubtful whether the species concerned should be included in deep sea fishes national biodiversity

Table 1. Species with more than 100 georeferenced records in the database. Minimum, maximum and mean depths.

\begin{tabular}{lcccc}
\hline Species & Records & Min depth (m) & Max depth (m) & Mean depth (m) \\
\hline Dibranchus atlanticus & 207 & 198 & 1,440 & 412 \\
Nezumia aequalis & 150 & 223 & 1,143 & 392 \\
Coelorinchus caelorhincus & 134 & 200 & 810 & 327 \\
Laemonema goodebeanorum & 126 & 223 & 777 & 348 \\
Chauliodus sloani & 125 & 191 & 4,151 & 433 \\
Synagrops bellus & 122 & 192 & 810 & 352 \\
Poecilopsetta inermis & 113 & 229 & 750 & 292 \\
Chlorophthalmus agassizi & 109 & 200 & 776 & 301 \\
Malacocephalus occidentalis & 106 & 200 & 801 & 314 \\
Neoscopelus macrolepidotus & 104 & 276 & 800 & 475 \\
Chaunax suttkusi & 103 & 223 & & 333 \\
\hline
\end{tabular}




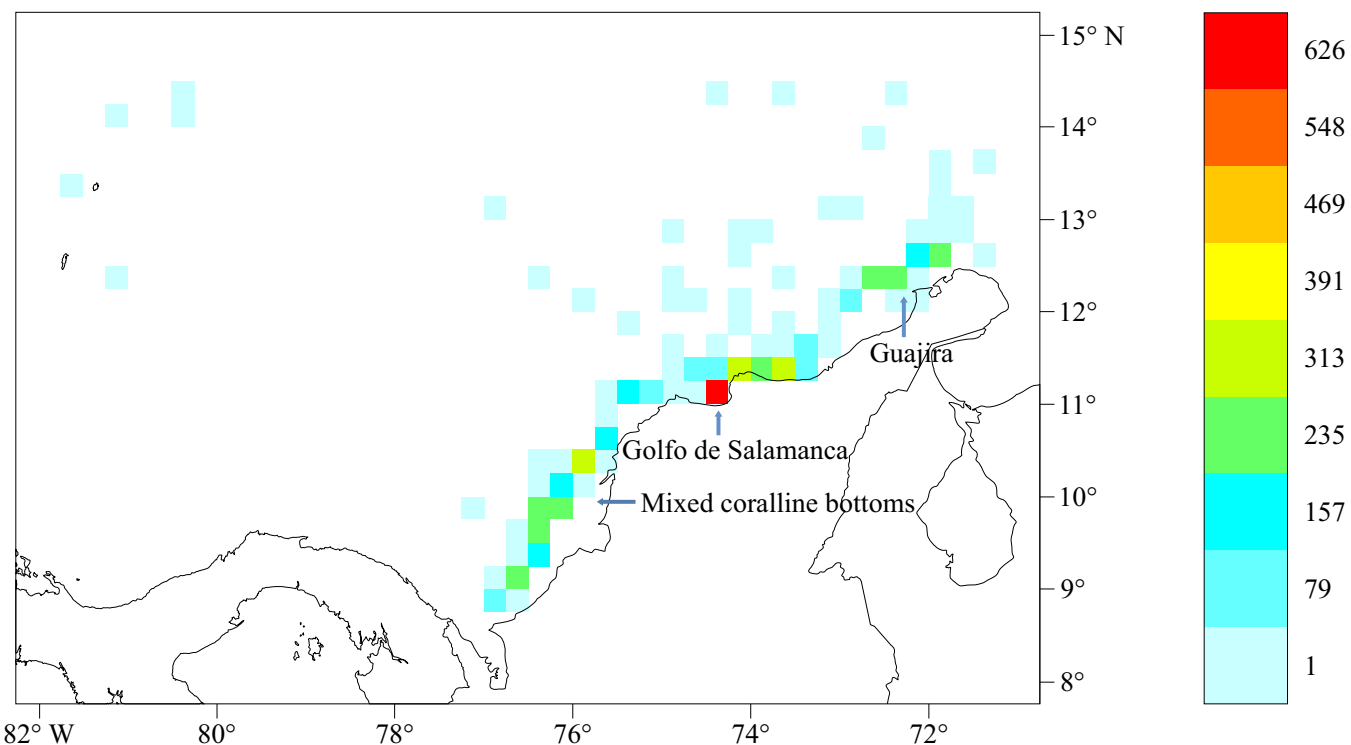

Figure 2. Spatial distribution of deep demersal fishes records in cells of 15 min by 15 min in the Colombian Caribbean Exclusive Economic Zone.

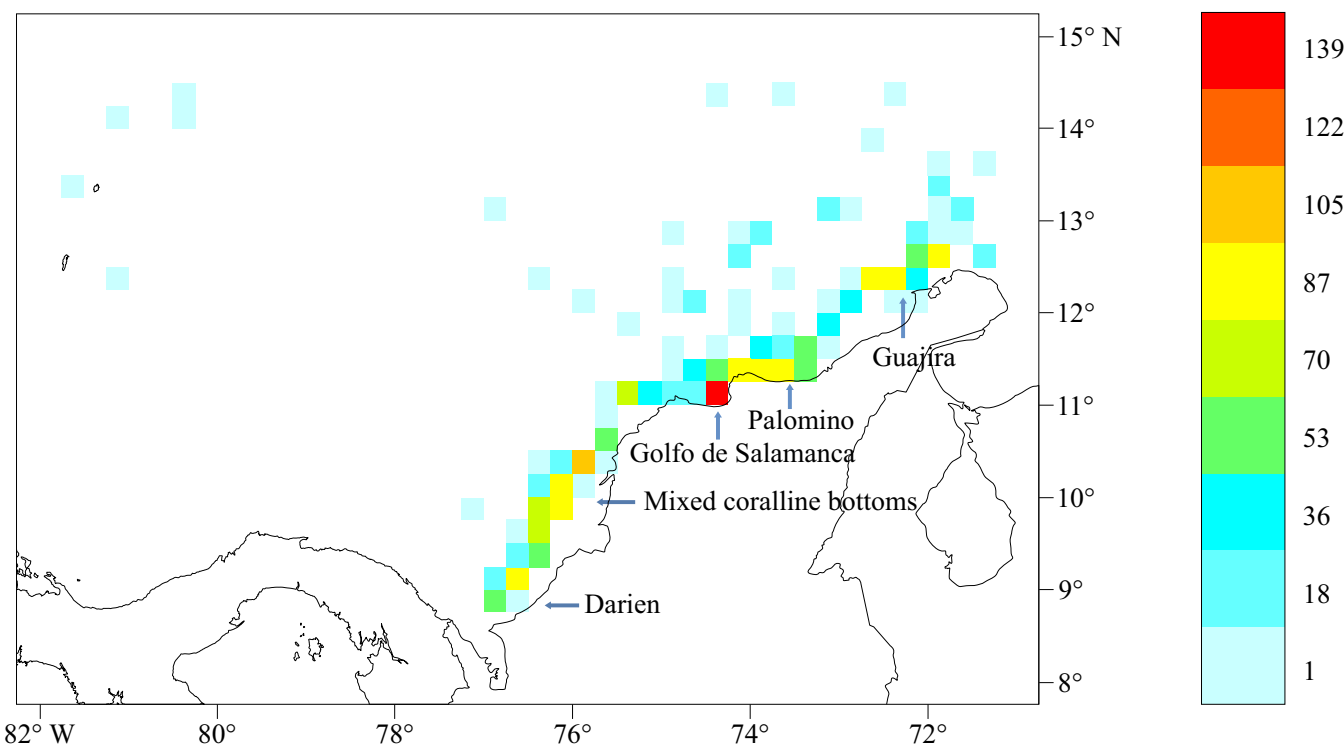

Figure 3 Observed richness of deep demersal fishes in cells of 15 min by $15 \mathrm{~min}$ in the Colombian Caribbean Exclusive Economic Zone.

lists, although their presence in waters of the archipelago that belong to Colombia is likely. Notice that Bolaños-Cubillos et al. (2015) and Acero et al. (2019) mention several of the species excluded here in their species lists for the San Andrés and Providencia archipelago.

The scarceness of surveys and samples east of the archipelago and in depths beyond 1,000 m 


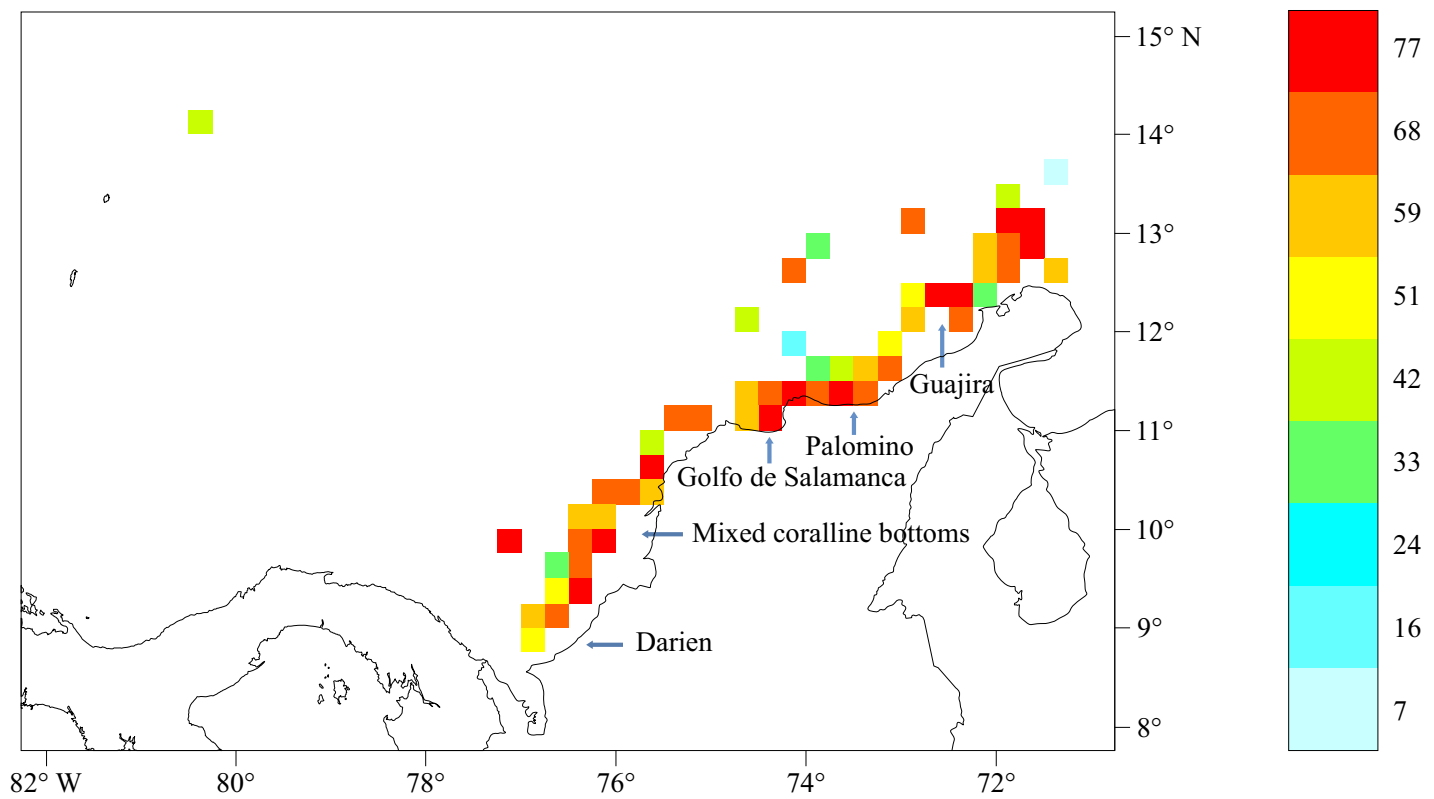

Figure 4 Percentage completeness of deep demersal fishes inventories in cells of 15 min by 15 min in the Colombian Caribbean Exclusive Economic Zone.

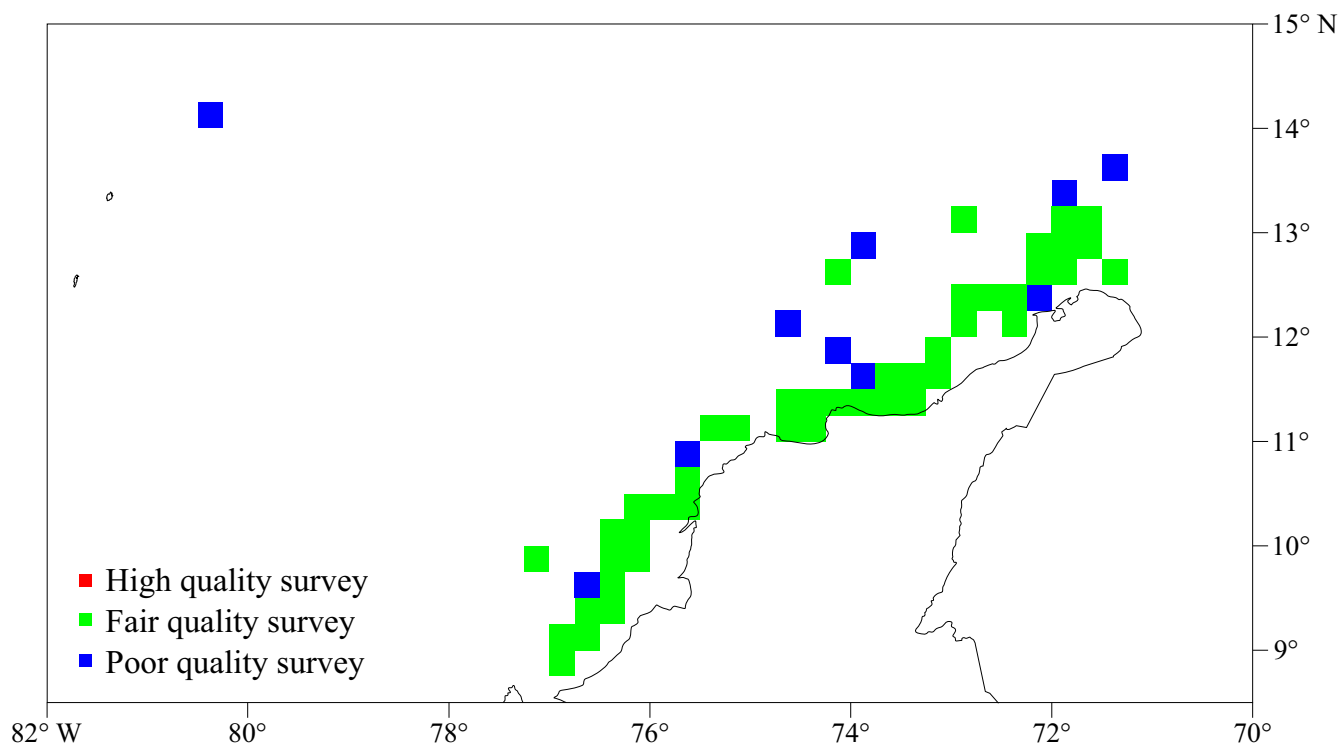

Figure 5 Survey quality status of deep demersal fishes in cells of $15 \mathrm{~min}$ by $15 \mathrm{~min}$ in the Colombian Caribbean Exclusive Economic Zone.

shown here clearly points to the need of increased survey efforts, including for those unexplored areas as well as renewed survey efforts in areas visited in the past, as even the upper slope that has received most records is at best 'fairly-sampled', according to the criteria here and at our 
spatial scale. Nevertheless, this study reveals a rich deep sea fish fauna taking into account that the 362 species in our database are a lower limit of species richness. Considering the values of percentage completeness and the current geographic coverage of surveys, more records for species already in the database, i.e., extension of their presently observed distribution, and more species currently not in the database, are to be expected in future campaigns.

The distribution of records with species follows the usual patterns for large diversity databases: few species with many records and many species with few records. Thus, $49.2 \%$ of the species (178 out of 362) show five or fewer records while just $3.0 \%$ of the species (11 out of 362) show more than one hundred records (Appendix 1). Interestingly Epigonus occidentalis (Goode and Bean 1896) and E. pandionis (Goode and Bean 1881), postulated as possible candidates for fishing due to their frequency in trawls (Páramo et al. 2017) do not belong to the most common species in the database but rank low to intermediary in terms of records (Appendix 1). Our time window spans decades while Páramo et al. (2017) are just snap shots of deep demersal fish presence and abundance in a limited depth range. This contrast highlights the usefulness and, indeed, a need for monitoring over long periods and extended areas if we are to understand the dynamics of ecosystems and the biology of the species.

In view of the results it is probably risky to postulate areas for conservation purposes. Observed richness suggests continental slope areas adjacent to the Gulf of Salamanca, Rosario Island archipelago (mixed coralline bottoms), slope areas to the north of Guajira Peninsula and Darien as locations of accumulation of species. Interestingly, the Gulf of Salamanca shelf $(<200$ $\mathrm{m})$, since here the slope $(>200 \mathrm{~m})$ is known to harbor significant numbers of elasmobranchs and bony fish species (García 2017b, 2018; but notice that García 2018 includes some slope records). The ecological and biological reasons for these findings are an open question worth investigating.

The only national natural park dedicated to deep sea biota in the Colombian Caribbean is the Parque Nacional Natural Corales de Profundidad established to protect deep sea coral species. The park includes soft and mixed bottoms from $34 \mathrm{~m}$ to 1,240 $\mathrm{m}$ depth. Using the module MRFinder of ModestR a species list was composed for the park resulting in 106 species with records inside the park area (Appendix 3). Thus, although not intentionally, Corales de Profundidad might also be protecting a significant fraction of deep demersal fishes in the Colombian Caribbean. The attractive effect of structures on fishes is wellknown, thus the presence of coral formations in the general area of the park is probably conducive of high species richness. Notice that cells in the park area and in their vicinity show high observed richness.

This research is one of the first steps in endeavors of studying the diversity of deep sea demersal fish species beyond the compilation of species' names. Much work remains to be done both for scientific and practical purposes with a focus on protecting Colombian Caribbean deep sea biodiversity as derived from this assessment.

\section{ACKNOWLEDGMENTS}

Comments by two anonimous reviewers helped to improve the manuscript.

\section{REFERENCES}

Acero A, Tavera JJ, Polanco A, BolañosCubillos N. 2019. Fish biodiversity in three northern islands of the seaflower biosphere reserve (Colombian Caribbean). Front Mar 
Sci. 6: 113. doi:10.3389/fmars.2019.00113

Acero A, Polo-Silva CJ, León G, Puentes V. 2018. First report of a sleeper shark (Somniosus sp.) in the southern Colombian Caribbean. J Appl Ichthyol. 34 (4): 981-983. doi:10.1111/ jai.13712

Bolaños-Cubillos N, Abril-Howard A, BentHooker H, Caldas JP, Acero A. 2015. Lista de peces conocidos del archipiélago de San Andrés, Providencia y Santa Catalina, reserva de biosfera Seaflower, Caribe occidental colombiano. Bol Investig Mar Cost. 44 (1): 127162. doi:10.25268/bimc.invemar.2015.44.1.24

Flanders Marine Institute. 2019. Maritime boundaries geodatabase: maritime boundaries and Exclusive Economic Zones (200NM), version 11. [accessed 2020 July]. https://www. marineregions.org/. doi:10.14284/386

FRICKE R, ESCHMEYER WN, VAN DER LAAN R. 2019, editors. Catalog of fishes: genera, species, references. [accessed 2020 June]. http:// researcharchive.calacademy.org/research/ ichthyology/catalog/fishcatmain.asp.

GARCíA CB. 2017a. Presence and distribution of Chimaeriformes in the Colombian Caribbean Sea. Pan Am J Aquat Sci. 12 (1): 85-88

GARCÍA CB. 2017b. What do we know about softbottom elasmobranch species richness in the Colombian Caribbean and of its spatial distribution? Reg Stud Mar Sci. 9: 62-68. doi:10.1016/j.rsma.2016.11.006

GARCíA CB. 2018. Spatial richness patterns of soft-bottom fish in the Colombian Caribbean continental shelf and slope. Acta Biol Colomb. 23 (1): 59-65. doi:10.15446/abc.v23n1.66530

García CB, Armenteras D. 2015. Atlas de la ictiofauna demersal de fondos blandos del Caribe continental colombiano: aproximación a su biodiversidad. Bogotá: Universidad Nacional de Colombia, Publicaciones Facultad de Ciencias. 765 p.

García-Roselló E, Guisande C, GonzálesDacosta J, Heine J, Pelayo-Villamil P, Manjarrés-Hernández A, VaAmonde A,
Granado-Lorencio C. 2013. ModestR: a software tool for managing and analyzing species distribution map databases. Ecography. 36: 1202-1207. doi:10.1111/j.16000587.2013.00374.x

[GBIF] Global Biodiversity InFormation FACILITY. 2020. GBIF Home Page. [accessed 2020 June]. https://www.gbif.org.

Grijalba-Bendeck M, Paramo J, WolfF M. 2019. Catch composition of deep-sea resources of commercial importance in the Colombian Caribbean. Rev Biol Mar Oceanogr. 54 (2): 188-197. doi:10.22370/rbmo.2019.54.2.1891

Guisande C, Heine J, GonzÁlez-DaCosta J, GARcíA-Roselló E. 2014. RWizard Software. http://www.ipez.es/RWizard. University of Vigo, Vigo.

Guisande C, Lobo JM. 2019. Discriminating well surveyed spatial units from exhaustive biodiversity databases. $\mathrm{R}$ package version. 2.0. https://cran.r-project.org/web/packages/ KnowBR.

Hortal J, de Bello F, Diniz-Filho FAA, LewinSHON TM, LoBo JM, LAdLE RJ. 2015. Seven shortfalls that beset large-scale knowledge of biodiversity. Annu Rev Ecol Evol Syst. 46: 523-549. doi:10.1146/annurev-ecolsys-112414054400

Loвo JM. 2008. Database records as a surrogate for sampling effort provide higher species richness estimations. Biodivers Conserv. 17: 873-881. doi:10.1007/s10531-008-9333-4

Lobo JM, Hortal J, Yelab JL, Millán A, SánCHEZ-FERNÁNDEZ D, GARCÍA-ROSELLÓ E, GONZÁlez-Dacosta J, HeINEe J, GonZÁlez-VILAS L, GuisAnde C. 2018. KnowBR: an application to map the geographical variation of survey effort and identify well-surveyed areas from biodiversity databases. Ecol Indic. 91: 241-248. doi:10.1016/j.ecolind.2018.03.077

PÁramo J, Fuentes D, Wiff R. 2017. Population structure and distribution of deep-water Cardinal Fish Epigonus occidentalis (Epigonidae) and Epigonus pandionis (Epigonidae) in the 
Colombian Caribbean Sea. J Ichthyol. 57 (3): 424-433. doi:10.1134/S0032945217030109

PÁramo J, Saint-Paul U. 2012. Deep-sea shrimps Aristaeomorpha foliacea and Pleoticus robustus (Crustacea: Penaeoidea) in the Colombian Caribbean Sea as a new potential fishing resource. J Mar Biol Assoc UK. 92 (4): 811-818. doi:10.1017/S0025315411001202

Páramo J, Saint-Paul U, Moreno F, Pacheco M, Almanza M, Rodríguez E, Ardila G, Borda C, BARRETo-GonzÁlez H. 2011. Crustáceos de profundidad en el Caribe colombiano como nuevo recurso pesquero. Informe Final Santa Marta: Universidad del Magdalena, Santa Marta.

Polanco A. 2015. Dynamics of the continental slope demersal fish community in the Colombian Caribbean-Deep-sea research in the Caribbean [PhD thesis]. Giessen: Justus-Liebig University Giessen, Bogotá: Universidad Nacional de Colombia. 192 p.

Polanco A, Acero A, Garrido M. 2010. Aportes a la biodiversidad íctica del Caribe colombiano. In: INVEMAR, editors. Biodiversidad del margen continental del Caribe colombiano. Serie de Publicaciones Especiales, Invemar. 20. p. 318-353.

Polanco A, Dueñas LF, León J, Puentes V. 2019. New records and update on the geographic distribution of the Bony-eared Assfish, Acanthonus armatus Günther, 1878 (Ophidiidae, Neobythitinae), in the Caribbean region. Check List. 15 (5): 767-772. doi:10. 15560/15.5.767

RATKOWSKI DA. 1990 Handbook of nonlinear regression models. New York: Marcel Dekker.
$241 \mathrm{p}$.

Roa-Varón A, SaAvedra LM, Acero A, Mejía LS. 2007. Nuevos registros de peces para el Caribe colombiano de los órdenes Myctophiformes, Polymiixiformes, Gadiformes, Ophidiiformes y Lophiiformes. Bol Investig Mar Cost. 36: 181-207. doi:10.25268/bimc.invemar.2007.36.0.206

Roa-Varón A, SaAvedra LM, Acero A, Mejía LS, NAVAS G. 2003. Nuevos registros de peces óseos para el Caribe colombiano de los órdenes Beryciformes, Zeiformes, Perciformes y Tetraodontiformes. Bol Investig Mar Cost. 32: 3-24. doi:10.25268/bimc.invemar.2003.32.0.257

SAAVEDRA-DíAz LM, ACERo A, NAVAS GR. 2000. Lenguados de la familia Paralichthyidae (Pisces: Pleuronectiformes) conocidos del incluyendo un nuevo registro para el área. Rev Acad Colomb Cienc Exactas Fis Nat. 24 (91): 295-310.

SaAVedra-Díaz LM, Roa-Varón A, Acero A, MeJía LS. 2004. Primeros registros ícticos en el talud superior del Caribe colombiano (órdenes Albuliformes, Anguilliformes, Stomiiformes, Ateleopodiformes, Aulopiformes y Pleuronectiformes). Bol Investig Mar Cost. 33: 159-183. doi:10.25268/bimc.invemar.2004.33.0.254

Soberón J, Jimenez R, Golubov J, KolefF P. 2007. Assessing completeness of biodiversity databases at different spatial scales. Ecography. 30: 152-160. doi:10.1111/j.0906-7590. 2007.04627.x

Ugland KI, Gray JS, Ellingsen KE. 2003. The species-accumulation curve and estimation of species richness. J Anim Ecol. 72: 888-897. doi:10.1046/j.1365-2656.2003.00748.x 


\section{APPENDIX 1}

\begin{tabular}{|c|c|c|c|c|c|c|c|}
\hline Class & Order & Family & Species & Records & Min depth & Max depth & Mean depth \\
\hline Actinopterygii & Anguilliformes & Chlopsidae & Robinsia catherinae & 1 & 300 & 300 & 300 \\
\hline Actinopterygii & Anguilliformes & Colocongridae & Coloconger meadi & 60 & 218 & 777 & 361 \\
\hline Actinopterygii & Anguilliformes & Congridae & Ariosoma balearicum & 3 & 532 & 857 & 706 \\
\hline Actinopterygii & Anguilliformes & Congridae & Bathycongrus bullisi & 26 & 229 & 366 & 286 \\
\hline Actinopterygii & Anguilliformes & Congridae & Bathycongrus vicinalis & 1 & 366 & 366 & 366 \\
\hline Actinopterygii & Anguilliformes & Congridae & Bathyuroconger vicinus & 9 & 515 & 732 & 627 \\
\hline Actinopterygii & Anguilliformes & Congridae & Japonoconger caribbeus & 22 & 269 & 549 & 358 \\
\hline Actinopterygii & Anguilliformes & Congridae & Parabathymyrus oregoni & 2 & 314 & 315 & 315 \\
\hline Actinopterygii & Anguilliformes & Congridae & Pseudophichthys splendens & 57 & 204 & 803 & 392 \\
\hline Actinopterygii & Anguilliformes & Congridae & Rhynchoconger flavus & 3 & 732 & 732 & 732 \\
\hline Actinopterygii & Anguilliformes & Congridae & Xenomystax austrinus & 23 & 458 & 732 & 509 \\
\hline Actinopterygii & Anguilliformes & Congridae & Xenomystax bidentatus & 16 & 296 & 698 & 405 \\
\hline Actinopterygii & Anguilliformes & Congridae & Xenomystax congroides & 18 & 210 & 352 & 237 \\
\hline Actinopterygii & Anguilliformes & Moringuidae & Neoconger mucronatus & 3 & 200 & 265 & 243 \\
\hline Actinopterygii & Anguilliformes & Muraenesocidae & Cynoponticus savanna & 2 & 746 & 746 & 746 \\
\hline Actinopterygii & Anguilliformes & Muraenidae & Gymnothorax conspersus & 9 & 256 & 307 & 262 \\
\hline Actinopterygii & Anguilliformes & Muraenidae & Gymnothorax polygonius & 2 & 200 & 203 & 202 \\
\hline Actinopterygii & Anguilliformes & Nemichthyidae & Avocettina infans & 3 & 350 & 445 & 360 \\
\hline Actinopterygii & Anguilliformes & Nemichthyidae & Labichthys carinatus & 1 & 940 & 940 & 940 \\
\hline Actinopterygii & Anguilliformes & Nemichthyidae & Nemichthys scolopaceus & 6 & 300 & 3,978 & 654 \\
\hline Actinopterygii & Anguilliformes & Nettastomatidae & Hoplunnis diomediana & 1 & 453 & 453 & 453 \\
\hline Actinopterygii & Anguilliformes & Nettastomatidae & Hoplunnis megista & 2 & 366 & 366 & 366 \\
\hline Actinopterygii & Anguilliformes & Nettastomatidae & Hoplunnis tenuis & 5 & 201 & 366 & 215 \\
\hline Actinopterygii & Anguilliformes & Nettastomatidae & Nettastoma melanura & 5 & 503 & 732 & 621 \\
\hline Actinopterygii & Anguilliformes & Nettastomatidae & Venefica procera & 3 & 613 & 860 & 834 \\
\hline Actinopterygii & Anguilliformes & Ophichthidae & Ophichthus cruentifer & 4 & 496 & 699 & 548 \\
\hline Actinopterygii & Anguilliformes & Ophichthidae & Ophichthus puncticeps & 4 & 295 & 900 & 448 \\
\hline Actinopterygii & Anguilliformes & Synaphobranchidae & Atractodenchelys phrix & 2 & 393 & 500 & 447 \\
\hline Actinopterygii & Anguilliformes & Synaphobranchidae & Dysommina rugosa & 2 & 365 & 450 & 429 \\
\hline Actinopterygii & Anguilliformes & Synaphobranchidae & Ilyophis brunneus & 8 & 515 & 1143 & 692 \\
\hline Actinopterygii & Anguilliformes & Synaphobranchidae & Synaphobranchus affinis & 1 & 732 & 732 & 732 \\
\hline Actinopterygii & Anguilliformes & Synaphobranchidae & Synaphobranchus oregoni & 8 & 265 & 515 & 301 \\
\hline Actinopterygii & Ateleopodiformes & Ateleopodidae & Ijimaia antillarum & 30 & 329 & 698 & 423 \\
\hline Actinopterygii & Ateleopodiformes & Ateleopodidae & Ijimaia loppei & 1 & 503 & 503 & 503 \\
\hline Actinopterygii & Aulopiformes & Bathysauridae & Bathysaurus mollis & 2 & 1,800 & 4,151 & 2,976 \\
\hline Actinopterygii & Aulopiformes & Chlorophthalmidae & Chlorophthalmus agassizi & 109 & 200 & 776 & 301 \\
\hline Actinopterygii & Aulopiformes & Chlorophthalmidae & Parasudis truculenta & 34 & 223 & 561 & 311 \\
\hline Actinopterygii & Aulopiformes & Ipnopidae & Bathypterois bigelowi & 38 & 223 & 940 & 456 \\
\hline Actinopterygii & Aulopiformes & Ipnopidae & Bathypterois grallator & 5 & 1627 & 4,151 & 2,435 \\
\hline Actinopterygii & Aulopiformes & Ipnopidae & Bathypterois phenax & 6 & 821 & 1,800 & 1,230 \\
\hline Actinopterygii & Aulopiformes & Ipnopidae & Bathypterois quadrifilis & 5 & 515 & 900 & 683 \\
\hline Actinopterygii & Aulopiformes & Ipnopidae & Bathypterois viridensis & 17 & 276 & 900 & 475 \\
\hline Actinopterygii & Aulopiformes & Ipnopidae & Bathytyphlops marionae & 1 & 1,251 & 1,251 & 1,251 \\
\hline Actinopterygii & Aulopiformes & Ipnopidae & Ipnops murrayi & 3 & 821 & 1,800 & 1,416 \\
\hline Actinopterygii & Aulopiformes & Notosudidae & Scopelosaurus smithii & 2 & 622 & 622 & 622 \\
\hline Actinopterygii & Aulopiformes & Synodontidae & Saurida brasiliensis & 5 & 270 & 613 & 427 \\
\hline Actinopterygii & Aulopiformes & Synodontidae & Saurida caribbaea & 9 & 200 & 303 & 219 \\
\hline Actinopterygii & Aulopiformes & Synodontidae & Saurida normani & 3 & 198 & 298 & 251 \\
\hline
\end{tabular}

Deep demersal fish species list (>200 m depth) of the Colombian Caribbean Sea with records and depth ranges. 
Appendix 1. Continued.

\begin{tabular}{|c|c|c|c|c|c|c|c|}
\hline Class & Order & Family & Species & Records & Min depth & Max depth & Mean depth \\
\hline Actinopterygii & Aulopiformes & Synodontidae & Synodus poeyi & 1 & 198 & 198 & 198 \\
\hline Actinopterygii & Batrachoidiformes & Batrachoididae & Porichthys plectrodon & 5 & 200 & 370 & 241 \\
\hline Actinopterygii & Beloniformes & Hemiramphidae & Hemiramphus balao & 2 & 276 & 803 & 406 \\
\hline Actinopterygii & Beryciformes & Anoplogastridae & Anoplogaster cornuta & 1 & 300 & 300 & 300 \\
\hline Actinopterygii & Beryciformes & Berycidae & Beryx decadactylus & 1 & 378 & 378 & 378 \\
\hline Actinopterygii & Beryciformes & Berycidae & Beryx splendens & 2 & 366 & 369 & 367 \\
\hline Actinopterygii & Beryciformes & Diretmidae & Diretmoides pauciradiatus & 9 & 200 & 732 & 268 \\
\hline Actinopterygii & Beryciformes & Diretmidae & Diretmus argenteus & 6 & 205 & 792 & 454 \\
\hline Actinopterygii & Beryciformes & Holocentridae & Ostichthys trachypoma & 6 & 200 & 274 & 236 \\
\hline Actinopterygii & Beryciformes & Trachichthyidae & Gephyroberyx darwinii & 8 & 300 & 375 & 322 \\
\hline Actinopterygii & Beryciformes & Trachichthyidae & Hoplostethus mediterraneus & 2 & 288 & 435 & 362 \\
\hline Actinopterygii & Beryciformes & Trachichthyidae & Hoplostethus occidentalis & 64 & 329 & 558 & 350 \\
\hline Actinopterygii & Cetomimiformes & Barbourisiidae & Barbourisia rufa & 1 & 1,080 & 1,080 & 1,080 \\
\hline Actinopterygii & Gadiformes & Bregmacerotidae & Bregmaceros atlanticus & 53 & 200 & 801 & 319 \\
\hline Actinopterygii & Gadiformes & Macrouridae & Bathygadus favosus & 21 & 515 & 1,440 & 758 \\
\hline Actinopterygii & Gadiformes & Macrouridae & Bathygadus macrops & 96 & 269 & 810 & 422 \\
\hline Actinopterygii & Gadiformes & Macrouridae & Bathygadus melanobranchus & 28 & 365 & 900 & 634 \\
\hline Actinopterygii & Gadiformes & Macrouridae & Cetonurus globiceps & 5 & 613 & 1,097 & 906 \\
\hline Actinopterygii & Gadiformes & Macrouridae & Coelorinchus caelorhincus & 134 & 200 & 810 & 327 \\
\hline Actinopterygii & Gadiformes & Macrouridae & Coelorinchus caribbaeus & 96 & 200 & 503 & 276 \\
\hline Actinopterygii & Gadiformes & Macrouridae & Coryphaenoides mexicanus & 12 & 198 & 1,296 & 884 \\
\hline Actinopterygii & Gadiformes & Macrouridae & Coryphaenoides zaniophorus & 25 & 518 & 940 & 636 \\
\hline Actinopterygii & Gadiformes & Macrouridae & Gadomus arcuatus & 17 & 477 & 1,080 & 665 \\
\hline Actinopterygii & Gadiformes & Macrouridae & Gadomus dispar & 4 & 457 & 622 & 490 \\
\hline Actinopterygii & Gadiformes & Macrouridae & Gadomus longifilis & 23 & 365 & 1,251 & 644 \\
\hline Actinopterygii & Gadiformes & Macrouridae & Hymenocephalus aterrimus & 1 & 576 & 576 & 576 \\
\hline Actinopterygii & Gadiformes & Macrouridae & Hymenocephalus billsam & 2 & 223 & 373 & 361 \\
\hline Actinopterygii & Gadiformes & Macrouridae & Hymenocephalus italicus & 91 & 269 & 940 & 368 \\
\hline Actinopterygii & Gadiformes & Macrouridae & Kuronezumia bubonis & 3 & 515 & 732 & 586 \\
\hline Actinopterygii & Gadiformes & Macrouridae & Malacocephalus laevis & 13 & 329 & 612 & 387 \\
\hline Actinopterygii & Gadiformes & Macrouridae & Malacocephalus occidentalis & 106 & 200 & 801 & 314 \\
\hline Actinopterygii & Gadiformes & Macrouridae & Nezumia aequalis & 150 & 223 & 1,143 & 392 \\
\hline Actinopterygii & Gadiformes & Macrouridae & Nezumia cyrano & 16 & 453 & 960 & 630 \\
\hline Actinopterygii & Gadiformes & Macrouridae & Nezumia suilla & 5 & 365 & 640 & 572 \\
\hline Actinopterygii & Gadiformes & Macrouridae & Sphagemacrurus grenadae & 6 & 684 & 1,143 & 779 \\
\hline Actinopterygii & Gadiformes & Macrouridae & Squalogadus modificatus & 4 & 1,080 & 1,251 & 1,139 \\
\hline Actinopterygii & Gadiformes & Macrouridae & Trachonurus sulcatus & 10 & 612 & 1,251 & 814 \\
\hline Actinopterygii & Gadiformes & Macrouridae & Trachonurus villosus & 3 & 515 & 814 & 714 \\
\hline Actinopterygii & Gadiformes & Macrouridae & Ventrifossa macropogon & 7 & 223 & 640 & 411 \\
\hline Actinopterygii & Gadiformes & Macrouridae & Ventrifossa mисосерhalus & 5 & 450 & 732 & 481 \\
\hline Actinopterygii & Gadiformes & Merlucciidae & Merluccius albidus & 47 & 219 & 662 & 297 \\
\hline Actinopterygii & Gadiformes & Merlucciidae & Steindachneria argentea & 66 & 200 & 770 & 298 \\
\hline Actinopterygii & Gadiformes & Moridae & Gadella imberbis & 32 & 223 & 801 & 374 \\
\hline Actinopterygii & Gadiformes & Moridae & Laemonema goodebeanorum & 126 & 223 & 777 & 348 \\
\hline Actinopterygii & Gadiformes & Moridae & Physiculus fulvus & 6 & 275 & 503 & 341 \\
\hline Actinopterygii & Gadiformes & Phycidae & Urophycis cirrata & 11 & 270 & 470 & 331 \\
\hline Actinopterygii & Lophiiformes & Antennariidae & Fowlerichthys radiosus & 1 & 192 & 192 & 192 \\
\hline Actinopterygii & Lophiiformes & Chaunacidae & Chaunax pictus & 57 & 270 & 810 & 417 \\
\hline Actinopterygii & Lophiiformes & Chaunacidae & Chaunax suttkusi & 103 & 223 & 801 & 333 \\
\hline Actinopterygii & Lophiiformes & Diceratiidae & Bufoceratias wedli & 4 & 405 & 493 & 478 \\
\hline Actinopterygii & Lophiiformes & Lophiidae & Lophiodes beroe & 2 & 512 & 512 & 512 \\
\hline
\end{tabular}


Appendix 1. Continued.

\begin{tabular}{|c|c|c|c|c|c|c|c|}
\hline Class & Order & Family & Species & Records & Min depth & Max depth & Mean depth \\
\hline Actinopterygii & Lophiiformes & Lophiidae & Lophiodes monodi & 7 & 313 & 520 & 346 \\
\hline Actinopterygii & Lophiiformes & Lophiidae & Lophiodes reticulatus & 11 & 200 & 500 & 240 \\
\hline Actinopterygii & Lophiiformes & Lophiidae & Lophius gastrophysus & 17 & 286 & 561 & 347 \\
\hline Actinopterygii & Lophiiformes & Lophiidae & Lophius piscatorius & 4 & 200 & 500 & 240 \\
\hline Actinopterygii & Lophiiformes & Melanocetidae & Melanocetus murrayi & 7 & 523 & 857 & 562 \\
\hline Actinopterygii & Lophiiformes & Ogcocephalidae & Dibranchus atlanticus & 207 & 198 & 1,440 & 412 \\
\hline Actinopterygii & Lophiiformes & Ogcocephalidae & Dibranchus tremendus & 6 & 1,006 & 1,463 & 1,107 \\
\hline Actinopterygii & Lophiiformes & Ogcocephalidae & Halieutichthys aculeatus & 20 & 286 & 295 & 291 \\
\hline Actinopterygii & Lophiiformes & Ogcocephalidae & Malthopsis gnoma & 12 & 280 & 491 & 301 \\
\hline Actinopterygii & Lophiiformes & Ogcocephalidae & Ogcocephalus declivirostris & 3 & 365 & 223 & 221 \\
\hline Actinopterygii & Lophiiformes & Ogcocephalidae & Ogcocephalus parvus & 6 & 270 & 360 & 308 \\
\hline Actinopterygii & Lophiiformes & Ogcocephalidae & Ogcocephalus pumilus & 1 & 290 & 290 & 290 \\
\hline Actinopterygii & Lophiiformes & Ogcocephalidae & Zalieutes mcgintyi & 29 & 201 & 498 & 246 \\
\hline Actinopterygii & Lophiiformes & Oneirodidae & Dolopichthys pullatus & 1 & 4,029 & 4,029 & 4,029 \\
\hline Actinopterygii & Lophiiformes & Thaumatichthyidae & Thaumatichthys binghami & 1 & 1,251 & 1,251 & 1,251 \\
\hline Actinopterygii & Myctophiformes & Myctophidae & Bolinichthys supralateralis & 26 & 195 & 792 & 361 \\
\hline Actinopterygii & Myctophiformes & Myctophidae & Dasyscopelus selenops & 2 & 275 & 520 & 396 \\
\hline Actinopterygii & Myctophiformes & Myctophidae & Diaphus adenomus & 1 & 567 & 567 & 567 \\
\hline Actinopterygii & Myctophiformes & Myctophidae & Diaphus bertelseni & 2 & 200 & 200 & 200 \\
\hline Actinopterygii & Myctophiformes & Myctophidae & Diaphus dumerilii & 26 & 200 & 727 & 299 \\
\hline Actinopterygii & Myctophiformes & Myctophidae & Diaphus effulgens & 2 & 223 & 365 & 330 \\
\hline Actinopterygii & Myctophiformes & Myctophidae & Diaphus garmani & 24 & 200 & 1,829 & 409 \\
\hline Actinopterygii & Myctophiformes & Myctophidae & Diaphus lucidus & 23 & 191 & 755 & 327 \\
\hline Actinopterygii & Myctophiformes & Myctophidae & Diaphus minax & 1 & 270 & 270 & 270 \\
\hline Actinopterygii & Myctophiformes & Myctophidae & Diaphus rafinesquii & 5 & 320 & 1,800 & 633 \\
\hline Actinopterygii & Myctophiformes & Myctophidae & Diaphus splendidus & 13 & 200 & 857 & 262 \\
\hline Actinopterygii & Myctophiformes & Myctophidae & Diaphus taaningi & 1 & 270 & 270 & 270 \\
\hline Actinopterygii & Myctophiformes & Myctophidae & Lampadena luminosa & 9 & 200 & 730 & 422 \\
\hline Actinopterygii & Myctophiformes & Myctophidae & Lepidophanes guentheri & 21 & 200 & 857 & 302 \\
\hline Actinopterygii & Myctophiformes & Myctophidae & Myctophum nitidulum & 16 & 200 & 727 & 340 \\
\hline Actinopterygii & Myctophiformes & Neoscopelidae & Neoscopelus macrolepidotus & 104 & 276 & 900 & 475 \\
\hline Actinopterygii & Myctophiformes & Neoscopelidae & Neoscopelus microchir & 23 & 223 & 803 & 372 \\
\hline Actinopterygii & Notacanthiformes & Halosauridae & Aldrovandia affinis & 5 & 515 & 1,097 & 559 \\
\hline Actinopterygii & Notacanthiformes & Halosauridae & Aldrovandia gracilis & 8 & 395 & 1,710 & 1,039 \\
\hline Actinopterygii & Notacanthiformes & Halosauridae & Halosaurus guentheri & 32 & 276 & 1,143 & 433 \\
\hline Actinopterygii & Notacanthiformes & Halosauridae & Halosaurus ovenii & 88 & 276 & 803 & 406 \\
\hline Actinopterygii & Notacanthiformes & Notacanthidae & Notacanthus chemnitzii & 1 & 724 & 724 & 724 \\
\hline Actinopterygii & Notacanthiformes & Notacanthidae & Polyacanthonotus merretti & 2 & 679 & 857 & 738 \\
\hline Actinopterygii & Ophidiiformes & Aphyonidae & Barathronus bicolor & 22 & 365 & 1,251 & 625 \\
\hline Actinopterygii & Ophidiiformes & Bythitidae & Calamopteryx robinsorum & 2 & 200 & 201 & 201 \\
\hline Actinopterygii & Ophidiiformes & Bythitidae & Cataetyx laticeps & 7 & 732 & 1,800 & 1,119 \\
\hline Actinopterygii & Ophidiiformes & Bythitidae & Diplacanthopoma brachysoma & 62 & 274 & 776 & 401 \\
\hline Actinopterygii & Ophidiiformes & Bythitidae & Saccogaster staigeri & 1 & 356 & 356 & 356 \\
\hline Actinopterygii & Ophidiiformes & Ophidiidae & Acanthonus armatus & 13 & 2,215 & 2,564 & 2,366 \\
\hline Actinopterygii & Ophidiiformes & Ophidiidae & Bassozetus robustus & 1 & 1,240 & 1,240 & 1,240 \\
\hline Actinopterygii & Ophidiiformes & Ophidiidae & Bathyonus laticeps & 2 & 1,627 & 2,983 & 2,531 \\
\hline Actinopterygii & Ophidiiformes & Ophidiidae & Benthocometes robustus & 4 & 223 & 303 & 287 \\
\hline Actinopterygii & Ophidiiformes & Ophidiidae & Dicrolene introniger & 37 & 395 & 1,296 & 715 \\
\hline Actinopterygii & Ophidiiformes & Ophidiidae & Lamprogrammus brunswigi & 2 & 1,317 & 1,317 & 1,317 \\
\hline Actinopterygii & Ophidiiformes & Ophidiidae & Lamprogrammus niger & 4 & 515 & 3978 & 1,105 \\
\hline Actinopterygii & Ophidiiformes & Ophidiidae & Lepophidium brevibarbe & 18 & 210 & 505 & 277 \\
\hline
\end{tabular}


Appendix 1. Continued.

\begin{tabular}{|c|c|c|c|c|c|c|c|}
\hline Class & Order & Family & Species & Records & Min depth & Max depth & Mean depth \\
\hline Actinopterygii & Ophidiiformes & Ophidiidae & Lepophidium cultratum & 2 & 210 & 210 & 210 \\
\hline Actinopterygii & Ophidiiformes & Ophidiidae & Lepophidium kallion & 1 & 219 & 219 & 219 \\
\hline Actinopterygii & Ophidiiformes & Ophidiidae & Lepophidium profundorum & 8 & 207 & 404 & 288 \\
\hline Actinopterygii & Ophidiiformes & Ophidiidae & Lepophidium robustum & 1 & 200 & 200 & 200 \\
\hline Actinopterygii & Ophidiiformes & Ophidiidae & Lepophidium zophochir & 3 & 210 & 210 & 210 \\
\hline Actinopterygii & Ophidiiformes & Ophidiidae & Luciobrotula corethromycter & 16 & 540 & 821 & 612 \\
\hline Actinopterygii & Ophidiiformes & Ophidiidae & Monomitopus agassizii & 47 & 365 & 1,251 & 528 \\
\hline Actinopterygii & Ophidiiformes & Ophidiidae & Neobythites gilli & 78 & 200 & 500 & 320 \\
\hline Actinopterygii & Ophidiiformes & Ophidiidae & Neobythites marginatus & 68 & 205 & 670 & 305 \\
\hline Actinopterygii & Ophidiiformes & Ophidiidae & Neobythites monocellatus & 4 & 229 & 334 & 257 \\
\hline Actinopterygii & Ophidiiformes & Ophidiidae & Neobythites ocellatus & 19 & 192 & 366 & 234 \\
\hline Actinopterygii & Ophidiiformes & Ophidiidae & Penopus microphthalmus & 1 & 1,006 & 1,006 & 1,006 \\
\hline Actinopterygii & Ophidiiformes & Ophidiidae & Xyelacyba myersi & 5 & 1,251 & 1,440 & 1,301 \\
\hline Actinopterygii & Osmeriformes & Alepocephalidae & Alepocephalus australis & 8 & 1,097 & 1,317 & 1,146 \\
\hline Actinopterygii & Osmeriformes & Alepocephalidae & Bathytroctes microlepis & 2 & 1,097 & 1,097 & 1,097 \\
\hline Actinopterygii & Osmeriformes & Alepocephalidae & Conocara macropterum & 10 & 821 & 1,463 & 1,125 \\
\hline Actinopterygii & Osmeriformes & Alepocephalidae & Leptoderma macrops & 1 & 690 & 690 & 690 \\
\hline Actinopterygii & Osmeriformes & Alepocephalidae & Narcetes stomias & 7 & 558 & 1,829 & 1,509 \\
\hline Actinopterygii & Osmeriformes & Alepocephalidae & Rouleina attrita & 4 & 686 & 1,271 & 828 \\
\hline Actinopterygii & Osmeriformes & Alepocephalidae & Talismania antillarum & 2 & 457 & 457 & 457 \\
\hline Actinopterygii & Osmeriformes & Alepocephalidae & Talismania homoptera & 5 & 515 & 640 & 593 \\
\hline Actinopterygii & Osmeriformes & Alepocephalidae & Xenodermichthys copei & 23 & 360 & 640 & 433 \\
\hline Actinopterygii & Osmeriformes & Argentinidae & Argentina brucei & 33 & 198 & 439 & 266 \\
\hline Actinopterygii & Osmeriformes & Argentinidae & Argentina striata & 72 & 200 & 500 & 266 \\
\hline Actinopterygii & Osmeriformes & Bathylagidae & Dolicholagus longirostris & 6 & 445 & 857 & 699 \\
\hline Actinopterygii & Osmeriformes & Microstomatidae & Xenophthalmichthys danae & 7 & 205 & 505 & 323 \\
\hline Actinopterygii & Osmeriformes & Opisthoproctidae & Opisthoproctus soleatus & 1 & 374 & 374 & 374 \\
\hline Actinopterygii & Perciformes & Acropomatidae & Caraibops trispinosus & 15 & 192 & 523 & 220 \\
\hline Actinopterygii & Perciformes & Acropomatidae & Synagrops bellus & 122 & 192 & 810 & 352 \\
\hline Actinopterygii & Perciformes & Acropomatidae & Verilus atlanticus & 10 & 192 & 496 & 250 \\
\hline Actinopterygii & Perciformes & Acropomatidae & Verilus pseudomicrolepis & 12 & 247 & 308 & 262 \\
\hline Actinopterygii & Perciformes & Acropomatidae & Verilus sordidus & 2 & 201 & 201 & 201 \\
\hline Actinopterygii & Perciformes & Ariommatidae & Ariomma bondi & 3 & 351 & 428 & 383 \\
\hline Actinopterygii & Perciformes & Ariommatidae & Ariomma melanum & 2 & 298 & 366 & 355 \\
\hline Actinopterygii & Perciformes & Bathyclupeidae & Bathyclupea argentea & 24 & 402 & 732 & 448 \\
\hline Actinopterygii & Perciformes & Bathyclupeidae & Bathyclupea schroederi & 16 & 205 & 810 & 387 \\
\hline Actinopterygii & Perciformes & Callionymidae & Synchiropus agassizii & 8 & 274 & 352 & 306 \\
\hline Actinopterygii & Perciformes & Callionymidae & Synchiropus dagmarae & 1 & 229 & 229 & 229 \\
\hline Actinopterygii & Perciformes & Caproidae & Antigonia capros & 37 & 198 & 505 & 240 \\
\hline Actinopterygii & Perciformes & Caproidae & Antigonia combatia & 51 & 192 & 520 & 243 \\
\hline Actinopterygii & Perciformes & Carangidae & Decapterus macarellus & 3 & 198 & 235 & 226 \\
\hline Actinopterygii & Perciformes & Carangidae & Decapterus tabl & 21 & 200 & 507 & 229 \\
\hline Actinopterygii & Perciformes & Carangidae & Selar crumenophthalmus & 7 & 246 & 2,195 & 575 \\
\hline Actinopterygii & Perciformes & Carangidae & Selene brownii & 3 & 200 & 504 & 403 \\
\hline Actinopterygii & Perciformes & Carangidae & Trachurus lathami & 2 & 207 & 207 & 207 \\
\hline Actinopterygii & Perciformes & Emmelichthyidae & Erythrocles monodi & 1 & 201 & 201 & 201 \\
\hline Actinopterygii & Perciformes & Epigonidae & Epigonus macrops & 13 & 200 & 914 & 613 \\
\hline Actinopterygii & Perciformes & Epigonidae & Epigonus occidentalis & 28 & 366 & 823 & 434 \\
\hline Actinopterygii & Perciformes & Epigonidae & Epigonus pandionis & 55 & 223 & 720 & 343 \\
\hline Actinopterygii & Perciformes & Gempylidae & Diplospinus multistriatus & 8 & 200 & 445 & 298 \\
\hline Actinopterygii & Perciformes & Gempylidae & Lepidocybium flavobrunneum & 2 & 1,251 & 1,271 & 1,261 \\
\hline
\end{tabular}


Appendix 1. Continued.

\begin{tabular}{|c|c|c|c|c|c|c|c|}
\hline Class & Order & Family & Species & Records & Min depth & Max depth & Mean depth \\
\hline Actinopterygii & Perciformes & Gempylidae & Nealotus tripes & 5 & 205 & 360 & 262 \\
\hline Actinopterygii & Perciformes & Gempylidae & Neoepinnula americana & 13 & 229 & 333 & 249 \\
\hline Actinopterygii & Perciformes & Gempylidae & Promethichthys prometheus & 17 & 298 & 807 & 386 \\
\hline Actinopterygii & Perciformes & Gempylidae & Ruvettus pretiosus & 3 & 396 & 558 & 461 \\
\hline Actinopterygii & Perciformes & Haemulidae & Haemulon aurolineatum & 2 & 286 & 286 & 286 \\
\hline Actinopterygii & Perciformes & Haemulidae & Haemulon boschmae & 2 & 286 & 295 & 291 \\
\hline Actinopterygii & Perciformes & Lutjanidae & Lutjanus vivanus & 1 & 324 & 324 & 324 \\
\hline Actinopterygii & Perciformes & Lutjanidae & Pristipomoides aquilonaris & 1 & 198 & 198 & 198 \\
\hline Actinopterygii & Perciformes & Lutjanidae & Pristipomoides macrophthalmus & 3 & 201 & 280 & 220 \\
\hline Actinopterygii & Perciformes & Mullidae & Upeneus parvus & 7 & 219 & 792 & 417 \\
\hline Actinopterygii & Perciformes & Opistognathidae & Lonchopisthus lemur & 32 & 200 & 300 & 242 \\
\hline Actinopterygii & Perciformes & Opistognathidae & Lonchopisthus micrognathus & 2 & 265 & 265 & 265 \\
\hline Actinopterygii & Perciformes & Percophidae & Bembrops anatirostris & 41 & 198 & 500 & 314 \\
\hline Actinopterygii & Perciformes & Percophidae & Bembrops gobioides & 6 & 360 & 540 & 439 \\
\hline Actinopterygii & Perciformes & Percophidae & Bembrops magnisquamis & 1 & 540 & 540 & 540 \\
\hline Actinopterygii & Perciformes & Percophidae & Bembrops ocellatus & 22 & 223 & 670 & 410 \\
\hline Actinopterygii & Perciformes & Percophidae & Bembrops quadrisella & 3 & 290 & 384 & 380 \\
\hline Actinopterygii & Perciformes & Priacanthidae & Heteropriacanthus cruentatus & 4 & 333 & 432 & 406 \\
\hline Actinopterygii & Perciformes & Sciaenidae & Protosciaena bathytatos & 12 & 240 & 512 & 340 \\
\hline Actinopterygii & Perciformes & Sciaenidae & Protosciaena trewavasae & 8 & 191 & 201 & 199 \\
\hline Actinopterygii & Perciformes & Scombrolabracidae & Scombrolabrax heterolepis & 1 & 396 & 396 & 396 \\
\hline Actinopterygii & Perciformes & Serranidae & Baldwinella aureorubens & 25 & 198 & 351 & 249 \\
\hline Actinopterygii & Perciformes & Serranidae & Baldwinella eos & 1 & 316 & 316 & 316 \\
\hline Actinopterygii & Perciformes & Serranidae & Bathyanthias cubensis & 7 & 192 & 280 & 209 \\
\hline Actinopterygii & Perciformes & Serranidae & Bathyanthias mexicanus & 1 & 300 & 300 & 300 \\
\hline Actinopterygii & Perciformes & Serranidae & Bullisichthys caribbaeus & 1 & 219 & 219 & 219 \\
\hline Actinopterygii & Perciformes & Serranidae & Hyporthodus flavolimbatus & 1 & 270 & 270 & 270 \\
\hline Actinopterygii & Perciformes & Serranidae & Hyporthodus nigritus & 2 & 200 & 333 & 267 \\
\hline Actinopterygii & Perciformes & Serranidae & Hyporthodus niveatus & 1 & 316 & 316 & 316 \\
\hline Actinopterygii & Perciformes & Serranidae & Plectranthias garrupellus & 2 & 200 & 200 & 200 \\
\hline Actinopterygii & Perciformes & Serranidae & Pronotogrammus martinicensis & 1 & 219 & 219 & 219 \\
\hline Actinopterygii & Perciformes & Serranidae & Serranus atrobranchus & 3 & 300 & 370 & 323 \\
\hline Actinopterygii & Perciformes & Sparidae & Pagrus pagrus & 1 & 207 & 207 & 207 \\
\hline Actinopterygii & Perciformes & Symphysanodontidae & Symphysanodon berryi & 3 & 200 & 280 & 216 \\
\hline Actinopterygii & Perciformes & Synagropidae & Parascombrops spinosus & 28 & 192 & 750 & 243 \\
\hline Actinopterygii & Perciformes & Trichiuridae & Benthodesmus simonyi & 15 & 223 & 659 & 302 \\
\hline Actinopterygii & Perciformes & Trichiuridae & Benthodesmus tenuis & 45 & 223 & 732 & 371 \\
\hline Actinopterygii & Perciformes & Trichiuridae & Lepidopus altifrons & 8 & 320 & 366 & 334 \\
\hline Actinopterygii & Perciformes & Trichiuridae & Lepidopus caudatus & 6 & 240 & 404 & 293 \\
\hline Actinopterygii & Perciformes & Trichiuridae & Trichiurus lepturus & 3 & 220 & 250 & 239 \\
\hline Actinopterygii & Pleuronectiformes & Bothidae & Chascanopsetta lugubris & 7 & 366 & 576 & 417 \\
\hline Actinopterygii & Pleuronectiformes & Bothidae & Monolene atrimana & 2 & 223 & 373 & 298 \\
\hline Actinopterygii & Pleuronectiformes & Bothidae & Monolene megalepis & 10 & 206 & 505 & 235 \\
\hline Actinopterygii & Pleuronectiformes & Bothidae & Trichopsetta caribbaea & 7 & 192 & 300 & 209 \\
\hline Actinopterygii & Pleuronectiformes & Bothidae & Trichopsetta ventralis & 2 & 198 & 252 & 225 \\
\hline Actinopterygii & Pleuronectiformes & Cynoglossidae & Symphurus diomedeanus & 2 & 290 & 316 & 303 \\
\hline Actinopterygii & Pleuronectiformes & Cynoglossidae & Symphurus ginsburgi & 11 & 296 & 491 & 348 \\
\hline Actinopterygii & Pleuronectiformes & Cynoglossidae & Symphurus hernandezi & 12 & 204 & 300 & 228 \\
\hline Actinopterygii & Pleuronectiformes & Cynoglossidae & Symphurus marginatus & 62 & 265 & 698 & 355 \\
\hline Actinopterygii & Pleuronectiformes & Cynoglossidae & Symphurus piger & 17 & 203 & 750 & 276 \\
\hline Actinopterygii & Pleuronectiformes & Cynoglossidae & Symphurus stigmosus & 1 & 274 & 274 & 274 \\
\hline
\end{tabular}


Appendix 1. Continued.

\begin{tabular}{|c|c|c|c|c|c|c|c|}
\hline Class & Order & Family & Species & Records & Min depth & Max depth & Mean depth \\
\hline Actinopterygii & Pleuronectiformes & Paralichthyidae & Ancylopsetta cycloidea & 9 & 192 & 269 & 207 \\
\hline Actinopterygii & Pleuronectiformes & Paralichthyidae & Citharichthys cornutus & 5 & 192 & 300 & 207 \\
\hline Actinopterygii & Pleuronectiformes & Pleuronectidae & Poecilopsetta beanii & 4 & 223 & 333 & 283 \\
\hline Actinopterygii & Pleuronectiformes & Pleuronectidae & Poecilopsetta inermis & 113 & 229 & 750 & 292 \\
\hline Actinopterygii & Polymixiiformes & Polymixiidae & Polymixia lowei & 72 & 200 & 940 & 282 \\
\hline Actinopterygii & Polymixiiformes & Polymixiidae & Polymixia nobilis & 7 & 366 & 512 & 412 \\
\hline Actinopterygii & Scorpaeniformes & Peristediidae & Peristedion ecuadorense & 34 & 223 & 810 & 374 \\
\hline Actinopterygii & Scorpaeniformes & Peristediidae & Peristedion gracile & 27 & 191 & 940 & 270 \\
\hline Actinopterygii & Scorpaeniformes & Peristediidae & Peristedion greyae & 53 & 265 & 803 & 334 \\
\hline Actinopterygii & Scorpaeniformes & Peristediidae & Peristedion imberbe & 1 & 219 & 219 & 219 \\
\hline Actinopterygii & Scorpaeniformes & Peristediidae & Peristedion longispatha & 38 & 223 & 766 & 414 \\
\hline Actinopterygii & Scorpaeniformes & Peristediidae & Peristedion miniatum & 42 & 229 & 720 & 291 \\
\hline Actinopterygii & Scorpaeniformes & Peristediidae & Peristedion truncatum & 18 & 223 & 731 & 348 \\
\hline Actinopterygii & Scorpaeniformes & Scorpaenidae & Neomerinthe beanorum & 3 & 200 & 300 & 260 \\
\hline Actinopterygii & Scorpaeniformes & Scorpaenidae & Phenacoscorpius nebris & 2 & 300 & 300 & 300 \\
\hline Actinopterygii & Scorpaeniformes & Scorpaenidae & Pontinus longispinis & 65 & 200 & 491 & 254 \\
\hline Actinopterygii & Scorpaeniformes & Scorpaenidae & Pontinus nematophthalmus & 9 & 200 & 698 & 288 \\
\hline Actinopterygii & Scorpaeniformes & Scorpaenidae & Pontinus rathbuni & 6 & 200 & 324 & 227 \\
\hline Actinopterygii & Scorpaeniformes & Sebastidae & Helicolenus dactylopterus & 3 & 293 & 662 & 366 \\
\hline Actinopterygii & Scorpaeniformes & Sebastidae & Trachyscorpia cristulata & 1 & 540 & 540 & 540 \\
\hline Actinopterygii & Scorpaeniformes & Setarchidae & Ectreposebastes imus & 5 & 400 & 732 & 550 \\
\hline Actinopterygii & Scorpaeniformes & Setarchidae & Setarches guentheri & 53 & 200 & 510 & 273 \\
\hline Actinopterygii & Scorpaeniformes & Triglidae & Bellator brachychir & 3 & 200 & 300 & 209 \\
\hline Actinopterygii & Scorpaeniformes & Triglidae & Bellator egretta & 2 & 200 & 203 & 201 \\
\hline Actinopterygii & Scorpaeniformes & Triglidae & Prionotus beanii & 1 & 219 & 219 & 219 \\
\hline Actinopterygii & Scorpaeniformes & Triglidae & Prionotus stearnsi & 10 & 213 & 351 & 273 \\
\hline Actinopterygii & Stephanoberyciformes & Gibberichthyidae & Gibberichthys pumilus & 3 & 720 & 732 & 728 \\
\hline Actinopterygii & Stephanoberyciformes & Stephanoberycidae & Stephanoberyx monae & 10 & 395 & 1,143 & 786 \\
\hline Actinopterygii & Stomiiformes & Gonostomatidae & Gonostoma atlanticum & 16 & 200 & 792 & 255 \\
\hline Actinopterygii & Stomiiformes & Gonostomatidae & Sigmops elongatus & 72 & 200 & 2341 & 452 \\
\hline Actinopterygii & Stomiiformes & Gonostomatidae & Triplophos hemingi & 28 & 300 & 732 & 424 \\
\hline Actinopterygii & Stomiiformes & Phosichthyidae & Ichthyococcus ovatus & 5 & 210 & 755 & 288 \\
\hline Actinopterygii & Stomiiformes & Phosichthyidae & Pollichthys mauli & 38 & 191 & 857 & 341 \\
\hline Actinopterygii & Stomiiformes & Phosichthyidae & Polymetme corythaeola & 19 & 275 & 857 & 487 \\
\hline Actinopterygii & Stomiiformes & Phosichthyidae & Polymetme thaeocoryla & 13 & 223 & 274 & 272 \\
\hline Actinopterygii & Stomiiformes & Phosichthyidae & Yarrella blackfordi & 26 & 283 & 1,143 & 625 \\
\hline Actinopterygii & Stomiiformes & Sternoptychidae & Argyripnus atlanticus & 1 & 260 & 260 & 260 \\
\hline Actinopterygii & Stomiiformes & Sternoptychidae & Argyropelecus aculeatus & 48 & 205 & 857 & 479 \\
\hline Actinopterygii & Stomiiformes & Sternoptychidae & Argyropelecus gigas & 1 & 540 & 540 & 540 \\
\hline Actinopterygii & Stomiiformes & Sternoptychidae & Maurolicus muelleri & 2 & 234 & 493 & 364 \\
\hline Actinopterygii & Stomiiformes & Sternoptychidae & Polyipnus asteroides & 50 & 205 & 567 & 338 \\
\hline Actinopterygii & Stomiiformes & Sternoptychidae & Polyipnus clarus & 4 & 205 & 265 & 259 \\
\hline Actinopterygii & Stomiiformes & Sternoptychidae & Polyipnus laternatus & 2 & 366 & 549 & 412 \\
\hline Actinopterygii & Stomiiformes & Sternoptychidae & Sternoptyx diaphana & 43 & 197 & 4,151 & 585 \\
\hline Actinopterygii & Stomiiformes & Sternoptychidae & Sternoptyx pseudobscura & 3 & 445 & 857 & 585 \\
\hline Actinopterygii & Stomiiformes & Stomiidae & Aristostomias grimaldii & 2 & 640 & 640 & 640 \\
\hline Actinopterygii & Stomiiformes & Stomiidae & Aristostomias xenostoma & 3 & 600 & 792 & 708 \\
\hline Actinopterygii & Stomiiformes & Stomiidae & Astronesthes macropogon & 9 & 225 & 1,710 & 412 \\
\hline Actinopterygii & Stomiiformes & Stomiidae & Chauliodus sloani & 125 & 191 & 4,151 & 433 \\
\hline Actinopterygii & Stomiiformes & Stomiidae & Eustomias schmidti & 2 & 450 & 857 & 586 \\
\hline Actinopterygii & Stomiiformes & Stomiidae & Heterophotus ophistoma & 3 & 640 & 732 & 671 \\
\hline
\end{tabular}


Appendix 1. Continued.

\begin{tabular}{|c|c|c|c|c|c|c|c|}
\hline Class & Order & Family & Species & Records & Min depth & Max depth & Mean depth \\
\hline Actinopterygii & Stomiiformes & Stomiidae & Malacosteus niger & 10 & 400 & 732 & 502 \\
\hline Actinopterygii & Stomiiformes & Stomiidae & Melanostomias macrophotus & 1 & 404 & 404 & 404 \\
\hline Actinopterygii & Stomiiformes & Stomiidae & Stomias affinis & 32 & 205 & 857 & 420 \\
\hline Actinopterygii & Stomiiformes & Stomiidae & Stomias longibarbatus & 3 & 225 & 755 & 358 \\
\hline Actinopterygii & Tetraodontiformes & Triacanthodidae & Hollardia hollardi & 6 & 329 & 558 & 350 \\
\hline Actinopterygii & Tetraodontiformes & Triacanthodidae & Parahollardia lineata & 1 & 369 & 369 & 369 \\
\hline Actinopterygii & Tetraodontiformes & Triacanthodidae & Parahollardia schmidti & 5 & 200 & 360 & 222 \\
\hline Actinopterygii & Zeiformes & Grammicolepididae & Grammicolepis brachiusculus & 8 & 324 & 662 & 442 \\
\hline Actinopterygii & Zeiformes & Grammicolepididae & Xenolepidichthys dalgleishi & 25 & 200 & 439 & 245 \\
\hline Actinopterygii & Zeiformes & Parazenidae & Cyttopsis rosea & 95 & 223 & 732 & 324 \\
\hline Actinopterygii & Zeiformes & Parazenidae & Parazen pacificus & 2 & 274 & 352 & 281 \\
\hline Actinopterygii & Zeiformes & Zeidae & Zenopsis conchifer & 2 & 270 & 298 & 284 \\
\hline Actinopterygii & Zeiformes & Zenionidae & Zenion hololepis & 72 & 200 & 540 & 385 \\
\hline Elasmobranchii & Carcharhiniformes & Carcharhinidae & Galeocerdo cuvier & 1 & 365 & 365 & 365 \\
\hline Elasmobranchii & Carcharhiniformes & Scyliorhinidae & Apristurus canutus & 2 & 530 & 777 & 592 \\
\hline Elasmobranchii & Carcharhiniformes & Scyliorhinidae & Apristurus parvipinnis & 2 & 719 & 719 & 719 \\
\hline Elasmobranchii & Carcharhiniformes & Scyliorhinidae & Apristurus riveri & 3 & 860 & 960 & 889 \\
\hline Elasmobranchii & Carcharhiniformes & Scyliorhinidae & Galeus arae & 4 & 256 & 512 & 374 \\
\hline Elasmobranchii & Carcharhiniformes & Scyliorhinidae & Galeus cadenati & 4 & 256 & 512 & 436 \\
\hline Elasmobranchii & Carcharhiniformes & Scyliorhinidae & Schroederichthys maculatus & 1 & 274 & 274 & 274 \\
\hline Elasmobranchii & Carcharhiniformes & Scyliorhinidae & Scyliorhinus boa & 19 & 223 & 540 & 298 \\
\hline Elasmobranchii & Carcharhiniformes & Scyliorhinidae & Scyliorhinus hesperius & 9 & 290 & 549 & 341 \\
\hline Elasmobranchii & Carcharhiniformes & Triakidae & Mustelus canis & 2 & 298 & 316 & 307 \\
\hline Elasmobranchii & Rajiformes & Anacanthobatidae & Schroederobatis americana & 51 & 307 & 803 & 475 \\
\hline Elasmobranchii & Rajiformes & Rajidae & Breviraja nigriventralis & 18 & 457 & 732 & 512 \\
\hline Elasmobranchii & Rajiformes & Rajidae & Breviraja spinosa & 4 & 540 & 612 & 575 \\
\hline Elasmobranchii & Rajiformes & Rajidae & Cruriraja rugosa & 7 & 365 & 732 & 449 \\
\hline Elasmobranchii & Rajiformes & Rajidae & Dactylobatus clarkii & 18 & 366 & 512 & 421 \\
\hline Elasmobranchii & Rajiformes & Rajidae & Dipturus bullisi & 9 & 201 & 334 & 302 \\
\hline Elasmobranchii & Rajiformes & Rajidae & Dipturus garricki & 3 & 283 & 307 & 301 \\
\hline Elasmobranchii & Rajiformes & Rajidae & Dipturus oregoni & 1 & 396 & 396 & 396 \\
\hline Elasmobranchii & Rajiformes & Rajidae & Dipturus teevani & 3 & 240 & 576 & 407 \\
\hline Elasmobranchii & Rajiformes & Rajidae & Fenestraja plutonia & 1 & 428 & 428 & 428 \\
\hline Elasmobranchii & Rajiformes & Rajidae & Fenestraja sinusmexicanus & 2 & 485 & 485 & 485 \\
\hline Elasmobranchii & Rajiformes & Rajidae & Gurgesiella atlantica & 34 & 223 & 698 & 498 \\
\hline Elasmobranchii & Squaliformes & Centrophoridae & Centrophorus granulosus & 4 & 200 & 732 & 333 \\
\hline Elasmobranchii & Squaliformes & Centrophoridae & Centrophorus squamosus & 1 & 670 & 670 & 670 \\
\hline Elasmobranchii & Squaliformes & Dalatiidae & Isistius brasiliensis & 1 & 621 & 621 & 621 \\
\hline Elasmobranchii & Squaliformes & Etmopteridae & Etmopterus bullisi & 1 & 274 & 274 & 274 \\
\hline Elasmobranchii & Squaliformes & Etmopteridae & Etmopterus carteri & 8 & 283 & 343 & 292 \\
\hline Elasmobranchii & Squaliformes & Etmopteridae & Etmopterus hillianus & 6 & 180 & 540 & 351 \\
\hline Elasmobranchii & Squaliformes & Etmopteridae & Etmopterus perryi & 22 & 283 & 375 & 297 \\
\hline Elasmobranchii & Squaliformes & Etmopteridae & Etmopterus pusillus & 1 & 288 & 288 & 288 \\
\hline Elasmobranchii & Squaliformes & Etmopteridae & Etmopterus schultzi & 43 & 269 & 823 & 422 \\
\hline Elasmobranchii & Squaliformes & Etmopteridae & Etmopterus virens & 25 & 288 & 503 & 331 \\
\hline Elasmobranchii & Squaliformes & Somniosidae & Somniosus cf microcephalus & 1 & 200 & 200 & 200 \\
\hline Elasmobranchii & Squaliformes & Squalidae & Squalus cubensis & 4 & 198 & 274 & 263 \\
\hline Elasmobranchii & Squatiniformes & Squatinidae & Squatina david & 2 & 198 & 305 & 252 \\
\hline Elasmobranchii & Torpediniformes & Torpedinidae & Tetronarce nobiliana & 6 & 292 & 369 & 329 \\
\hline Holocephali & Chimaeriformes & Chimaeridae & Chimaera cubana & 1 & 234 & 234 & 234 \\
\hline Holocephali & Chimaeriformes & Chimaeridae & Hydrolagus alberti & 27 & 223 & 1,143 & 477 \\
\hline
\end{tabular}


Appendix 1. Continued.

\begin{tabular}{|c|c|c|c|c|c|c|c|}
\hline Class & Order & Family & Species & Records & Min depth & Max depth & Mean depth \\
\hline Holocephali & Chimaeriformes & Chimaeridae & Hydrolagus mirabilis & 3 & 720 & 1,296 & 995 \\
\hline Holocephali & Chimaeriformes & Rhinochimaeridae & Neoharriotta carri & 14 & 288 & 485 & 329 \\
\hline Holocephali & Chimaeriformes & Rhinochimaeridae & Rhinochimaera atlantica & 2 & 914 & 917 & 916 \\
\hline Myxini & Myxiniformes & Myxinidae & Eptatretus aceroi & 2 & 705 & 705 & 705 \\
\hline Myxini & Myxiniformes & Myxinidae & Eptatretus ancon & 8 & 476 & 670 & 513 \\
\hline Myxini & Myxiniformes & Myxinidae & Eptatretus wayuu & 5 & 300 & 303 & 301 \\
\hline Myxini & Myxiniformes & Myxinidae & Myxine mccoskeri & 72 & 269 & 801 & 446 \\
\hline Myxini & Myxiniformes & Myxinidae & Myxine robinsorum & 2 & 783 & 821 & 793 \\
\hline
\end{tabular}

\section{APPENDIX 2}

\begin{tabular}{|c|c|c|c|}
\hline Class & Order & Family & Species \\
\hline Actinopterygii & Anguilliformes & Congridae & Acromycter atlanticus \\
\hline Actinopterygii & Anguilliformes & Congridae & Bathycongrus thysanochilus \\
\hline Actinopterygii & Anguilliformes & Congridae & Rhynchoconger gracilior \\
\hline Actinopterygii & Anguilliformes & Nettastomatidae & Hoplunnis similis \\
\hline Actinopterygii & Aulopiformes & Giganturidae & Gigantura chuni \\
\hline Actinopterygii & Aulopiformes & Ipnopidae & Bathytyphlops sewelli \\
\hline Actinopterygii & Aulopiformes & Paralepididae & Lestrolepis intermedia \\
\hline Actinopterygii & Aulopiformes & Paralepididae & Stemonosudis rothschildi \\
\hline Actinopterygii & Batrachoidiformes & Batrachoididae & Porichthys bathoiketes \\
\hline Actinopterygii & Ophidiiformes & Ophidiidae & Lepophidium entomelan \\
\hline Actinopterygii & Ophidiiformes & Ophidiidae & Lepophidium marmoratum \\
\hline Actinopterygii & Ophidiiformes & Ophidiidae & Lepophidium staurophor \\
\hline Actinopterygii & Osmeriformes & Argentinidae & Argentina stewarti \\
\hline Actinopterygii & Osmeriformes & Argentinidae & Glossanodon pygmaeus \\
\hline Actinopterygii & Perciformes & Apogonidae & Paroncheilus affinis \\
\hline Actinopterygii & Perciformes & Labridae & Decodon puellaris \\
\hline Actinopterygii & Perciformes & Lutjanidae & Rhomboplites aurorubens \\
\hline Actinopterygii & Perciformes & Percophidae & Bembrops macromma \\
\hline Actinopterygii & Perciformes & Serranidae & Serranus phoebe \\
\hline Actinopterygii & Perciformes & Uranoscopidae & Kathetostoma cubana \\
\hline Actinopterygii & Pleuronectiformes & Paralichthyidae & Ancylopsetta microctenus \\
\hline Actinopterygii & Pleuronectiformes & Paralichthyidae & Citharichthys dinoceros \\
\hline Actinopterygii & Stomiiformes & Sternoptychidae & Sonoda megalophthalma \\
\hline Actinopterygii & Stomiiformes & Stomiidae & Echiostoma barbatum \\
\hline Actinopterygii & Stomiiformes & Stomiidae & Eustomias acinosus \\
\hline Elasmobranchii & Rajiformes & Rajidae & Breviraja mouldi \\
\hline Elasmobranchii & Rajiformes & Rajidae & Fenestraja ishiyamai \\
\hline Elasmobranchii & Rajiformes & Rajidae & Leucoraja garmani \\
\hline Elasmobranchii & Squaliformes & Etmopteridae & Etmopterus robinsi \\
\hline Elasmobranchii & Squaliformes & Squalidae & Squalus mitsukurii \\
\hline Myxini & Myxiniformes & Myxinidae & Eptatretus caribbeaus \\
\hline Myxini & Myxiniformes & Myxinidae & Eptatretus multidens \\
\hline
\end{tabular}

Species excluded from data base because records fall outside the Colombian Caribbean Exclusive Economic Zone. 


\section{APPENDIX 3}

\begin{tabular}{|c|c|c|c|c|}
\hline Class & Order & Family & Genus & Species \\
\hline Actinopterygii & Anguilliformes & Colocongridae & Coloconger & Coloconger meadi \\
\hline Actinopterygii & Anguilliformes & Congridae & Bathycongrus & Bathycongrus bullisi \\
\hline Actinopterygii & Anguilliformes & Congridae & Pseudophichthys & Pseudophichthys splendens \\
\hline Actinopterygii & Anguilliformes & Congridae & Xenomystax & Xenomystax congroides \\
\hline Actinopterygii & Aulopiformes & Chlorophthalmidae & Chlorophthalmus & Chlorophthalmus agassizi \\
\hline Actinopterygii & Aulopiformes & Chlorophthalmidae & Parasudis & Parasudis truculenta \\
\hline Actinopterygii & Aulopiformes & Ipnopidae & Bathypterois & Bathypterois bigelowi \\
\hline Actinopterygii & Aulopiformes & Synodontidae & Saurida & Saurida brasiliensis \\
\hline Actinopterygii & Beryciformes & Trachichthyidae & Hoplostethus & Hoplostethus occidentalis \\
\hline Actinopterygii & Gadiformes & Bregmacerotidae & Bregmaceros & Bregmaceros atlanticus \\
\hline Actinopterygii & Gadiformes & Macrouridae & Bathygadus & Bathygadus macrops \\
\hline Actinopterygii & Gadiformes & Macrouridae & Coelorinchus & Coelorinchus caelorhincus \\
\hline Actinopterygii & Gadiformes & Macrouridae & Coelorinchus & Coelorinchus caribbaeus \\
\hline Actinopterygii & Gadiformes & Macrouridae & Gadomus & Gadomus arcuatus \\
\hline Actinopterygii & Gadiformes & Macrouridae & Hymenocephalus & Hymenocephalus billsam \\
\hline Actinopterygii & Gadiformes & Macrouridae & Hymenocephalus & Hymenocephalus italicus \\
\hline Actinopterygii & Gadiformes & Macrouridae & Malacocephalus & Malacocephalus laevis \\
\hline Actinopterygii & Gadiformes & Macrouridae & Malacocephalus & Malacocephalus occidentalis \\
\hline Actinopterygii & Gadiformes & Macrouridae & Nezumia & Nezumia aequalis \\
\hline Actinopterygii & Gadiformes & Macrouridae & Ventrifossa & Ventrifossa macropogon \\
\hline Actinopterygii & Gadiformes & Merlucciidae & Steindachneria & Steindachneria argentea \\
\hline Actinopterygii & Gadiformes & Moridae & Gadella & Gadella imberbis \\
\hline Actinopterygii & Gadiformes & Moridae & Laemonema & Laemonema goodebeanorum \\
\hline Actinopterygii & Gadiformes & Moridae & Physiculus & Physiculus fulvus \\
\hline Actinopterygii & Lophiiformes & Chaunacidae & Chaunax & Chaunax pictus \\
\hline Actinopterygii & Lophiiformes & Chaunacidae & Chaunax & Chaunax suttkusi \\
\hline Actinopterygii & Lophiiformes & Lophiidae & Lophiodes & Lophiodes monodi \\
\hline Actinopterygii & Lophiiformes & Ogcocephalidae & Dibranchus & Dibranchus atlanticus \\
\hline Actinopterygii & Lophiiformes & Ogcocephalidae & Halieutichthys & Halieutichthys aculeatus \\
\hline Actinopterygii & Lophiiformes & Ogcocephalidae & Malthopsis & Malthopsis gnoma \\
\hline Actinopterygii & Lophiiformes & Ogcocephalidae & Ogcocephalus & Ogcocephalus declivirostris \\
\hline Actinopterygii & Myctophiformes & Myctophidae & Dasyscopelus & Dasyscopelus selenops \\
\hline Actinopterygii & Myctophiformes & Myctophidae & Diaphus & Diaphus effulgens \\
\hline Actinopterygii & Myctophiformes & Myctophidae & Diaphus & Diaphus garmani \\
\hline Actinopterygii & Myctophiformes & Myctophidae & Diaphus & Diaphus lucidus \\
\hline Actinopterygii & Myctophiformes & Neoscopelidae & Neoscopelus & Neoscopelus macrolepidotus \\
\hline Actinopterygii & Myctophiformes & Neoscopelidae & Neoscopelus & Neoscopelus microchir \\
\hline Actinopterygii & Notacanthiformes & Halosauridae & Halosaurus & Halosaurus guentheri \\
\hline Actinopterygii & Notacanthiformes & Halosauridae & Halosaurus & Halosaurus ovenii \\
\hline Actinopterygii & Notacanthiformes & Notacanthidae & Notacanthus & Notacanthus chemnitzii \\
\hline Actinopterygii & Ophidiiformes & Bythitidae & Calamopteryx & Calamopteryx robinsorum \\
\hline Actinopterygii & Ophidiiformes & Bythitidae & Diplacanthopoma & Diplacanthopoma brachysoma \\
\hline Actinopterygii & Ophidiiformes & Ophidiidae & Benthocometes & Benthocometes robustus \\
\hline Actinopterygii & Ophidiiformes & Ophidiidae & Monomitopus & Monomitopus agassizii \\
\hline Actinopterygii & Ophidiiformes & Ophidiidae & Neobythites & Neobythites gilli \\
\hline Actinopterygii & Ophidiiformes & Ophidiidae & Neobythites & Neobythites marginatus \\
\hline Actinopterygii & Osmeriformes & Argentinidae & Argentina & Argentina striata \\
\hline Actinopterygii & Perciformes & Acropomatidae & Caraibops & Caraibops trispinosus \\
\hline
\end{tabular}

Deep demersal species ( $>200 \mathrm{~m}$ depth) recorded inside the Parque Nacional Natural National Natural Park Corales de Profundidad in the Colombian Caribbean. 
Appendix 3. Continued.

\begin{tabular}{|c|c|c|c|c|}
\hline Class & Order & Family & Genus & Species \\
\hline Actinopterygii & Perciformes & Acropomatidae & Synagrops & Synagrops bellus \\
\hline Actinopterygii & Perciformes & Acropomatidae & Verilus & Verilus atlanticus \\
\hline Actinopterygii & Perciformes & Acropomatidae & Verilus & Verilus pseudomicrolepis \\
\hline Actinopterygii & Perciformes & Bathyclupeidae & Bathyclupea & Bathyclupea argentea \\
\hline Actinopterygii & Perciformes & Bathyclupeidae & Bathyclupea & Bathyclupea schroederi \\
\hline Actinopterygii & Perciformes & Caproidae & Antigonia & Antigonia capros \\
\hline Actinopterygii & Perciformes & Caproidae & Antigonia & Antigonia combatia \\
\hline Actinopterygii & Perciformes & Carangidae & Decapterus & Decapterus tabl \\
\hline Actinopterygii & Perciformes & Carangidae & Selar & Selar crumenophthalmus \\
\hline Actinopterygii & Perciformes & Epigonidae & Epigonus & Epigonus macrops \\
\hline Actinopterygii & Perciformes & Epigonidae & Epigonus & Epigonus occidentalis \\
\hline Actinopterygii & Perciformes & Epigonidae & Epigonus & Epigonus pandionis \\
\hline Actinopterygii & Perciformes & Lutjanidae & Pristipomoides & Pristipomoides macrophthalmus \\
\hline Actinopterygii & Perciformes & Opistognathidae & Lonchopisthus & Lonchopisthus lemur \\
\hline Actinopterygii & Perciformes & Percophidae & Bembrops & Bembrops anatirostris \\
\hline Actinopterygii & Perciformes & Percophidae & Bembrops & Bembrops gobioides \\
\hline Actinopterygii & Perciformes & Percophidae & Bembrops & Bembrops ocellatus \\
\hline Actinopterygii & Perciformes & Percophidae & Bembrops & Bembrops quadrisella \\
\hline Actinopterygii & Perciformes & Serranidae & Baldwinella & Baldwinella aureorubens \\
\hline Actinopterygii & Perciformes & Serranidae & Bathyanthias & Bathyanthias cubensis \\
\hline Actinopterygii & Perciformes & Serranidae & Hyporthodus & Hyporthodus nigritus \\
\hline Actinopterygii & Perciformes & Symphysanodontidae & Symphysanodon & Symphysanodon berryi \\
\hline Actinopterygii & Perciformes & Trichiuridae & Benthodesmus & Benthodesmus simonyi \\
\hline Actinopterygii & Perciformes & Trichiuridae & Benthodesmus & Benthodesmus tenuis \\
\hline Actinopterygii & Pleuronectiformes & Bothidae & Monolene & Monolene atrimana \\
\hline Actinopterygii & Pleuronectiformes & Bothidae & Monolene & Monolene megalepis \\
\hline Actinopterygii & Pleuronectiformes & Cynoglossidae & Symphurus & Symphurus marginatus \\
\hline Actinopterygii & Pleuronectiformes & Paralichthyidae & Ancylopsetta & Ancylopsetta cycloidea \\
\hline Actinopterygii & Pleuronectiformes & Pleuronectidae & Poecilopsetta & Poecilopsetta beanii \\
\hline Actinopterygii & Pleuronectiformes & Pleuronectidae & Poecilopsetta & Poecilopsetta inermis \\
\hline Actinopterygii & Polymixiiformes & Polymixiidae & Polymixia & Polymixia lowei \\
\hline Actinopterygii & Scorpaeniformes & Peristediidae & Peristedion & Peristedion ecuadorense \\
\hline Actinopterygii & Scorpaeniformes & Peristediidae & Peristedion & Peristedion gracile \\
\hline Actinopterygii & Scorpaeniformes & Peristediidae & Peristedion & Peristedion greyae \\
\hline Actinopterygii & Scorpaeniformes & Peristediidae & Peristedion & Peristedion longispatha \\
\hline Actinopterygii & Scorpaeniformes & Peristediidae & Peristedion & Peristedion truncatum \\
\hline Actinopterygii & Scorpaeniformes & Scorpaenidae & Neomerinthe & Neomerinthe beanorum \\
\hline Actinopterygii & Scorpaeniformes & Scorpaenidae & Pontinus & Pontinus nematophthalmus \\
\hline Actinopterygii & Scorpaeniformes & Setarchidae & Setarches & Setarches guentheri \\
\hline Actinopterygii & Stomiiformes & Phosichthyidae & Polymetme & Polymetme corythaeola \\
\hline Actinopterygii & Stomiiformes & Phosichthyidae & Polymetme & Polymetme thaeocoryla \\
\hline Actinopterygii & Stomiiformes & Sternoptychidae & Argyripnus & Argyripnus atlanticus \\
\hline Actinopterygii & Stomiiformes & Sternoptychidae & Argyropelecus & Argyropelecus aculeatus \\
\hline Actinopterygii & Stomiiformes & Sternoptychidae & Argyropelecus & Argyropelecus gigas \\
\hline Actinopterygii & Stomiiformes & Sternoptychidae & Maurolicus & Maurolicus muelleri \\
\hline Actinopterygii & Stomiiformes & Sternoptychidae & Polyipnus & Polyipnus asteroides \\
\hline Actinopterygii & Stomiiformes & Sternoptychidae & Polyipnus & Polyipnus laternatus \\
\hline Actinopterygii & Stomiiformes & Stomiidae & Chauliodus & Chauliodus sloani \\
\hline Actinopterygii & Tetraodontiformes & Triacanthodidae & Parahollardia & Parahollardia schmidti \\
\hline Actinopterygii & Zeiformes & Grammicolepididae & Xenolepidichthys & Xenolepidichthys dalgleishi \\
\hline Actinopterygii & Zeiformes & Parazenidae & Cyttopsis & Cyttopsis rosea \\
\hline
\end{tabular}


Appendix 3. Continued.

\begin{tabular}{lllll}
\hline Class & \multicolumn{1}{c}{ Order } & \multicolumn{1}{c}{ Family } & Genus & Species \\
\hline Actinopterygii & Zeiformes & Zenionidae & Zenion & Zenion hololepis \\
Elasmobranchii & Carcharhiniformes & Scyliorhinidae & Scyliorhinus & Scyliorhinus boa \\
Elasmobranchii & Rajiformes & Rajidae & Breviraja & Breviraja spinosa \\
Elasmobranchii & Rajiformes & Rajidae & Dipturus & Dipturus bullisi \\
Elasmobranchii & Rajiformes & Rajidae & Gurgesiella & Gurgesiella atlantica \\
Holocephali & Chimaeriformes & Chimaeridae & Hydrolagus & Hydrolagus alberti \\
Myxini & Myxiniformes & Myxinidae & Myxine & Myxine mccoskeri \\
\hline
\end{tabular}

